\title{
Superconductivity from repulsion: Variational results for the two-dimensional Hubbard model in the limit of weak interaction
}

\author{
Dionys Baeriswyl* \\ Department of Physics, University of Fribourg, Chemin du Musée 3, CH-1700 Fribourg, Switzerland, \\ and International Institute of Physics, 59078-400 Natal-RN, Brazil
}

(Received 12 September 2018; revised manuscript received 28 May 2019; published 27 June 2019)

\begin{abstract}
The two-dimensional Hubbard model is studied for small values of the interaction strength ( $U$ of the order of the hopping amplitude $t$ ), using a variational ansatz well suited for this regime. The wave function, a refined Gutzwiller ansatz, has a BCS mean-field state with $d$-wave symmetry as its reference state. Superconducting order is found for densities $n<1$ (but not for $n=1$ ). This resolves a discrepancy between results obtained with the functional renormalization group, which do predict superconducting order for small values of $U$, and numerical simulations, which did not find superconductivity for $U \lesssim 4 t$. Both the gap parameter and the order parameter have a domelike shape as a function of $n$ with a maximum for $n \approx 0.8$. Expectation values for the energy, the particle number, and the superconducting order parameter are calculated using a linked-cluster expansion up to second order in $U$. In this way large systems (millions of sites) can be readily treated and well converged results are obtained. A big size is indeed required to see that the gap becomes very small at half filling and probably tends to zero in the thermodynamic limit, whereas away from half filling a finite asymptotic limit is reached. For a lattice of a given size the order parameter becomes finite only above a minimal coupling strength $U_{c}$. This threshold value decreases steadily with increasing system size, which indicates that superconductivity exists for arbitrarily small $U$ for an infinite system. For moderately large systems the size dependence is quite irregular, due to variations in level spacings at the Fermi energy. The fluctuations die out if the gap parameter spans several level spacings.
\end{abstract}

DOI: 10.1103/PhysRevB.99.235152

\section{INTRODUCTION}

The Hubbard model was introduced more than fifty years ago by Anderson [1], Gutzwiller [2], Hubbard [3], and Kanamori [4] for discussing magnetic ordering in narrowband materials. Later on the model was used for describing the Mott metal-insulator transition [5] and it even served as a microscopic basis for Landau's Fermi-liquid theory of ${ }^{3} \mathrm{He}$ [6]. A dramatic upsurge of interest set in when Anderson postulated that the Hubbard model on a square lattice embodies the essentials of cuprate superconductors, by reproducing both the insulating antiferromagnetic phase of the parent compounds and the superconducting phase observed upon doping [7]. Still today, it is not clear to what extent Anderson's postulate is corroborated by experiments.

That superconductivity can arise from purely repulsive interactions was already shown in 1965 by Kohn and Luttinger [8]. Using perturbation theory for a continuum model with weak short-range and purely repulsive interaction, they found superconductivity for an unconventional symmetry of the order parameter. During the last thirty years a lot of effort has been spent to find out whether pairing by repulsion is also realized in the two-dimensional Hubbard model, for positive values of the on-site coupling parameter $U$.

For large values of $U$ there is a general consensus that superconductivity, preferentially with $d$-wave symmetry, exists

*dionys.baeriswyl@unifr.ch for a density close to one particle per site, i.e., for a nearly half-filled band $[9,10]$. Variational methods have been particularly helpful for estimating the energy gap and the superconducting order parameter, and for studying the competition between antiferromagnetism and superconductivity [11,12]. Cluster extensions of dynamical mean-field theory have also found evidence for ( $d$-wave) pairing for intermediate to large values of $U$ [13], but different schemes yield rather different results [14].

For small values of $U$, the method of the functional renormalization group has been particularly helpful for tracking the instabilities of the two-dimensional Hubbard model [15]. Initially, superconducting instabilities were detected through divergences of the effective pairing interaction in the normal phase [16-19]. More recently, techniques were developed to continue the procedure into the superconducting phase, either by using "partial bosonization" [20] or by combining the scaling in the normal phase with mean-field theory in the ordered phase [21]. While calculations based on the functional renormalization group consistently predict superconductivity ( $d$-wave pairing close to half filling), they cannot provide quantitative results for the energy gap or for the order parameter.

Variational calculations do make quantitative predictions, but, besides from being to some extent biased, they usually rely on Monte Carlo sampling, therefore they are limited to modest system sizes (typically $20 \times 20$ sites for a square lattice) and suffer from statistical uncertainties. This should not be a problem if the energy gap due to superconductivity 
is large enough, i.e., for moderate to large values of $U$. However, for small values of $U$, where variational Monte Carlo calculations are unable to find any evidence for superconductivity, one has to worry about the reliability of the method.

In Gutzwiller's celebrated variational ansatz [2] an operator acts on an uncorrelated state to reduce double occupancy. A simple modification refines this ansatz by adding an operator involving the kinetic energy [22,23]. The refined wave function is particularly well suited for treating the small $U$ limit [23-25]. Superconductivity of the two-dimensional Hubbard model has been explored by applying variational Monte Carlo to this ansatz [26-28].

In the present paper a slightly modified version of the refined Gutzwiller wave function is worked out perturbatively. Our method is restricted to a relatively small region of coupling strengths, $0.35 t \lesssim U \lesssim 1.2 t$, where $t$ is the hopping amplitude between nearest-neighbor sites. Nevertheless, the results are clear-cut, especially so because very large system sizes can be treated easily (millions of sites). We choose as the "initial" uncorrelated state a BCS ansatz with a simple $d$-wave symmetry, where pairing occurs between nearest-neighbor sites. The Hubbard model of course also embodies other orderings, such as antiferromagnetism and $p$ wave superconductivity [29], or superconductivity with more complicated nodal structures than the most simple $p$ - and $d$-wave order parameters [30], but here we limit ourselves to $d$-wave pairing.

We do find superconductivity away from half filling (but not at half filling), with a largely increased order parameter as compared to results obtained with the standard Gutzwiller ansatz. The $U$ dependence of the gap parameter is consistent with a power law. This dependence differs from what has been found in random-phase approximation (RPA)-type theories for spin-fluctuation exchange. We also find that superconductivity is not necessarily produced by a lowering of potential energy, but depending on filling it may also be due to a lowering of the "kinetic energy" (the expectation value of the hopping term). Therefore non-BCS features are not necessarily a privilege of the large $U$ region of the Hubbard model, i.e., of the doped Mott insulator, but they may also show up already for small values of $U$, i.e., in the "itinerant part" of the phase diagram.

The paper is organized as follows. The variational ansatz is introduced in Sec. II, where also the linked-cluster expansion is explained. Section III presents the variational ground-state energy for the normal state (vanishing gap parameter), in comparison to a straightforward perturbative expansion in powers of $U$. In Sec. IV the formalism is applied to the superconducting state (finite gap parameter). Some details about the minimization procedure are also given. Section V deals with the condensation energy and the delicate balance between kinetic- and potential-energy lowering. Results for the gap parameter are discussed in detail in Sec. VI, including its "domelike" dependence on particle density as well as an unconventional dependence on $U$. Section VII introduces the superconducting order parameter and shows that it has a similar domelike shape as the gap parameter. The dependence on system size is examined in Sec. VIII. In Sec. IX the results obtained with the refined wave function are compared with those deduced with the conventional Gutzwiller ansatz. The paper is summarized in Sec. X, where also a few problems are listed which could be studied in the future. Some technicalities are discussed in Appendixes A and C. In Appendix B an effective model is treated which has a superconducting meanfield ground state with $d$-wave symmetry.

\section{VARIATIONAL APPROACH}

\section{A. Hamiltonian}

We consider the (repulsive) Hubbard Hamiltonian $H=$ $H_{0}+H_{\text {int }}$, with nearest-neighbor hopping,

$$
H_{0}=-t \sum_{\left\langle\mathbf{R}, \mathbf{R}^{\prime}\right\rangle} \sum_{\sigma}\left(c_{\mathbf{R} \sigma}^{\dagger} c_{\mathbf{R}^{\prime} \sigma}+\text { H.c. }\right)
$$

and on-site repulsion,

$$
H_{\text {int }}=U \sum_{\mathbf{R}} n_{\mathbf{R} \uparrow} n_{\mathbf{R} \downarrow}
$$

where the operator $c_{\mathbf{R} \sigma}^{\dagger}\left(c_{\mathbf{R} \sigma}\right)$ creates (annihilates) an electron at site $\mathbf{R}$ with spin projection $\sigma=\uparrow$ or $\downarrow$, and $n_{\mathbf{R} \sigma}:=c_{\mathbf{R} \sigma}^{\dagger} c_{\mathbf{R} \sigma}$. We restrict ourselves to a square lattice with $N_{s}=L \times L$ sites and a lattice constant 1 . The hopping amplitude is taken as the unit of energy, i.e., we put $t=1$. In Fourier space we have

$$
\begin{aligned}
H_{0} & =-\sum_{\mathbf{k} \sigma} \varepsilon_{\mathbf{k}} c_{\mathbf{k} \sigma}^{\dagger} c_{\mathbf{k} \sigma}, \\
H_{\text {int }} & =\frac{U}{N_{s}} \sum_{\mathbf{k}_{1}, \ldots, \mathbf{k}_{4}} \delta\left(\mathbf{k}_{1}-\mathbf{k}_{2}+\mathbf{k}_{3}-\mathbf{k}_{4}\right) c_{\mathbf{k}_{1} \uparrow}^{\dagger} c_{\mathbf{k}_{2} \uparrow} c_{\mathbf{k}_{3} \downarrow}^{\dagger} c_{\mathbf{k}_{4} \downarrow},
\end{aligned}
$$

where the Kronecker symbol $\delta(\mathbf{k})$ is equal to 1 if $\mathbf{k}$ is a reciprocal-lattice vector and 0 otherwise. The tight-binding spectrum is given by

$$
\varepsilon_{\mathbf{k}}=-2\left(\cos k_{x}+\cos k_{y}\right) .
$$

The identity

$$
\delta(\mathbf{k})=\frac{1}{N_{s}} \sum_{\mathbf{R}} e^{i \mathbf{k} \cdot \mathbf{R}}
$$

will be extensively used later on.

\section{B. Variational ansatz}

The (standard) Gutzwiller ansatz reads

$$
\left|\Psi_{G}\right\rangle:=e^{-\eta D}\left|\Psi_{0}\right\rangle,
$$

where $D=\sum_{\mathbf{R}} n_{\mathbf{R} \uparrow} n_{\mathbf{R} \downarrow}$ measures the number of doubly occupied sites, $\eta$ is a variational parameter, and $\left|\Psi_{0}\right\rangle$ is the ground state of $H_{0}$ (the filled Fermi sea). To deal with ordering phenomena, such as antiferromagnetism or superconductivity, one uses, instead of $\left|\Psi_{0}\right\rangle$, the ground state $\left|\Psi_{m}\right\rangle$ of a symmetry-breaking mean-field Hamiltonian $H_{m}$ as the reference state.

The ansatz (6) has been widely adopted in the limit $\eta \rightarrow \infty$, where double occupancy is completely suppressed and the Hubbard Hamiltonian can be replaced by its large $U$ limit, which corresponds to the $t-J$ model $[11,12]$. The case of finite $\eta$ with a superconducting reference state has been treated both numerically, using Monte Carlo sampling [31], and partly analytically, by a perturbative expansion [32]. 
Unfortunately, the ansatz (6) has its weaknesses. Both the energy and the momentum distribution are at odds with exact results in one dimension, where the Gutzwiller wave function can be analyzed exactly [33]. A remedy was proposed already in the early 1980s in terms of an additional prefactor which strengthens the correlations between doubly occupied and empty sites ("doublon-holon binding") [34]. This additional term turned out to be important for intermediate to large values of $U$, but not for small $U$. Variational Monte Carlo calculations with this modified wave function yield a superconducting ground state in the intermediate to strong-coupling regime and for not too large doping, with clear deviations from BCS behavior [35,36]. A Jastrow factor producing long-range charge-charge correlations [37] has also been proposed. It can lead to long-range order in the absence of a symmetrybreaking field [38,39]. Another way of improving the ansatz (6) is to modify the reference state $\left|\Psi_{m}\right\rangle$. For instance, instead of using two parameters for a superconducting mean-field state (the gap parameter and the "chemical potential"), one can independently vary the BCS amplitudes $u_{\mathbf{k}}$ and in this way introduce a huge number of variational parameters (of the order of $N_{s}$ ) [25]. It has also been proposed to incorporate a "backflow term" to improve the accuracy and to account for the kinetic exchange for large values of $U$ [40].

Our ansatz reads

$$
|\Psi\rangle:=e^{-\tau H_{m}} e^{-\eta D}\left|\Psi_{m}\right\rangle,
$$

where $\left|\Psi_{m}\right\rangle$ is the ground state of the mean-field Hamiltonian $H_{m}$ (with energy eigenvalue $E_{m}$ ) and $\tau$ is an additional variational parameter. Equation (7) differs slightly from the ansatz used in previous (variational Monte Carlo) studies [26,27], where the "kinetic energy" $H_{0}$ was used in the exponent. The choice of $H_{m}$ instead of $H_{0}$ is very convenient for a perturbative evaluation of expectation values, as will become clear below. One could even argue that this choice is natural because symmetry breaking is introduced by replacing the eigenstate of $H_{0}$ by that of $H_{m}$, therefore it is quite logical to replace also $H_{0}$ by $H_{m}$.

\section{Linked cluster expansion}

When Gutzwiller introduced his wave function [2], he adopted the linked cluster expansion for calculating expectation values. A detailed derivation has been given later by Horsch and Fulde [41]. Here we show that the expansion can also be used for our ansatz (7).

The variational parameters $\eta, \tau$ plus those defining the mean-field state $\left|\Psi_{m}\right\rangle$ are determined by minimizing the energy

$$
\bar{E}=\frac{\langle\Psi|H| \Psi\rangle}{\langle\Psi \mid \Psi\rangle}
$$

for a given average number of particles

$$
\bar{N}=\frac{\langle\Psi|N| \Psi\rangle}{\langle\Psi \mid \Psi\rangle},
$$

where $N=\sum_{\mathbf{k} \sigma} c_{\mathbf{k} \sigma}^{\dagger} c_{\mathbf{k} \sigma}$. Equation (7) can be written as

$$
|\Psi\rangle:=e^{-\tau E_{m}} e^{-\eta D(\tau)}\left|\Psi_{m}\right\rangle,
$$

where we have introduced the notation

$$
\mathcal{O}(\tau):=e^{-\tau H_{m}} \mathcal{O} e^{\tau H_{m}}
$$

for any operator $\mathcal{O}$. Correspondingly, we have

$$
\bar{E}=\frac{\left\langle\Psi_{m}\left|e^{-\eta D(-\tau)} H e^{-\eta D(\tau)}\right| \Psi_{m}\right\rangle}{\left\langle\Psi_{m}\left|e^{-\eta D(-\tau)} e^{-\eta D(\tau)}\right| \Psi_{m}\right\rangle},
$$

and, because of the linked-cluster theorem,

$$
\bar{E}=\left\langle\Psi_{m}\left|e^{-\eta D(-\tau)} H e^{-\eta D(\tau)}\right| \Psi_{m}\right\rangle_{c},
$$

where $c$ means that only those diagrams have to be taken into account where either both exponentials are connected to the operator $H$ or one of the two is connected to $H$ and the two exponential operators are connected to each other. We carry the expansion out to second order in $\eta$ for the hopping term $H_{0}$ and to first order for the interaction $H_{\text {int }}=U D$. This is justified because the optimized correlation parameter $\eta$ is linear in $U$ for $U \rightarrow 0$ and hence the second-order contribution to the interaction part would be proportional to $U^{3}$, i.e., negligible at this order of the expansion [42]. We find

$$
\begin{aligned}
\bar{E} \approx & \langle H\rangle-2 \eta\langle H D(\tau)\rangle_{c} \\
& +\eta^{2}\left[\left\langle H_{0} D^{2}(\tau)\right\rangle_{c}+\left\langle D(-\tau) H_{0} D(\tau)\right\rangle_{c}\right],
\end{aligned}
$$

where we have introduced the notation

$$
\langle\mathcal{O}\rangle:=\left\langle\Psi_{m}|\mathcal{O}| \Psi_{m}\right\rangle
$$

for averages with respect to the mean-field ground state. The average particle number is calculated in the same way, and we obtain

$$
\begin{aligned}
\bar{N} \approx & \langle N\rangle-2 \eta\langle N D(\tau)\rangle_{c} \\
& +\eta^{2}\left[\left\langle N D^{2}(\tau)\right\rangle_{c}+\langle D(-\tau) N D(\tau)\rangle_{c}\right] .
\end{aligned}
$$

If $\left|\Psi_{m}\right\rangle$ is an eigenstate of $N$ all the connected terms vanish and $\bar{N}=\langle N\rangle$. This is the case for $\left|\Psi_{m}\right\rangle=\left|\Psi_{0}\right\rangle$ or for an antiferromagnetic reference state, but not for a BCS state, for which Eq. (16) together with the constraint of a fixed $\bar{N}$ yields a nontrivial relation between the variational parameters. For $\left|\Psi_{m}\right\rangle=\left|\Psi_{0}\right\rangle$ the contributions $\left\langle H_{0} D(\tau)\right\rangle_{c}$ and $\left\langle H_{0} D^{2}(\tau)\right\rangle_{c}$ also vanish.

For a fixed mean-field state $\left|\Psi_{m}\right\rangle$ and a fixed parameter $\tau$, the energy (14) is easily minimized with respect to $\eta$. Obviously $\eta$ has to be small enough, otherwise the limitation to second order is no longer a good approximation. How small? A simple argument can be given by looking at the problem of two particles on two sites, for which the Gutzwiller ansatz is exact. One readily finds that the second-order expansion corresponds to the replacement

$$
1-e^{-\eta} \rightarrow \eta-\frac{\eta^{2}}{2}
$$

For $\eta<0.5$ this approximation is very good, better than 95\%. This simple estimate agrees with an explicit comparison between a full evaluation of the Gutzwiller ansatz (variational Monte Carlo) and the second-order expansion for the onedimensional Peierls-Hubbard model, showing good agreement for $\eta \lesssim 0.7$ [43]. In the present paper we limit ourselves to the region where $\eta$ does not exceed values of the order of 0.6. This criterion implies that $U$ should be lower than about 
A
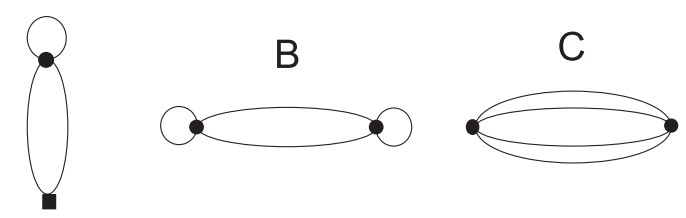

FIG. 1. First-order diagrams for the expansion (14). Dots represent two-particle vertices, squares single-particle vertices.

1.2 for the full ansatz (7), while the Gutzwiller wave function (6) admits $U$ values up to 3.5 .

\section{NORMAL STATE}

We discuss first the symmetric case, $H_{m}=H_{0},\left|\Psi_{m}\right\rangle=$ $\left|\Psi_{0}\right\rangle$. The various terms of the expansion (14) are readily calculated by Wick decomposition. The zeroth-order term is given by

$$
\langle H\rangle=2 \sum_{\mathbf{k}} \varepsilon_{\mathbf{k}} P_{\mathbf{k}}+\frac{N_{s}}{4} U n^{2},
$$

where

$$
P_{\mathbf{k}}=\left\langle c_{\mathbf{k} \sigma}^{\dagger} c_{\mathbf{k} \sigma}\right\rangle
$$

is the Fermi function (which does not depend on $\sigma$ for a nondegenerate state, where all levels are either fully occupied or unoccupied), and

$$
n=\frac{\bar{N}}{N_{s}}=\frac{2}{N_{s}} \sum_{\mathbf{k}} P_{\mathbf{k}}
$$

is the particle density. It is convenient to introduce also the Fermi function of holes,

$$
Q_{\mathbf{k}}=\left\langle c_{\mathbf{k} \sigma} c_{\mathbf{k} \sigma}^{\dagger}\right\rangle .
$$

The first-order contributions are illustrated by diagrams in Fig. 1. The lines represent

$$
\left\langle c_{\mathbf{k} \sigma}^{\dagger} c_{\mathbf{k} \sigma}(\tau)\right\rangle=e^{-\tau\left|\xi_{\mathbf{k}}\right|} P_{\mathbf{k}}
$$

or

$$
\left\langle c_{\mathbf{k} \sigma} c_{\mathbf{k} \sigma}^{\dagger}(\tau)\right\rangle=e^{-\tau\left|\xi_{\mathbf{k}}\right|} Q_{\mathbf{k}},
$$

where $\xi_{\mathbf{k}}=\varepsilon_{\mathbf{k}}-\varepsilon_{F}$ is the single-particle spectrum measured with respect to the Fermi energy $\varepsilon_{F}$. Thus the parameter $\tau^{-1}$ acts like a soft energy cutoff, which renders correlation effects strongest close to the Fermi surface. We know already that the contribution $A$, which represents the term $\left\langle H_{0} D(\tau)\right\rangle_{c}$, has to vanish. This follows also from the fact that diagram $A$ involves the factor $P_{\mathrm{k}} Q_{\mathrm{k}}(=0)$. The same is true for the contribution $B$, which therefore also vanishes. To evaluate the contribution $C$, we transform the threefold momentum sum into a single sum over lattice sites using Eq. (5). We obtain

$$
\left\langle H_{\text {int }} D(\tau)\right\rangle_{c}=N_{s} U \sum_{\mathbf{R}}[P(\mathbf{R}, \tau) Q(\mathbf{R}, \tau)]^{2},
$$

where $(X=P$ or $Q)$

$$
X(\mathbf{R}, \tau):=\frac{1}{N_{s}} \sum_{\mathbf{k}} e^{i \mathbf{k} \cdot \mathbf{R}} e^{-\tau\left|\xi_{\mathbf{k}}\right|} X_{\mathbf{k}}
$$
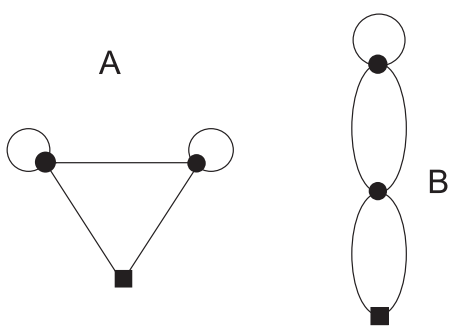

C

FIG. 2. Second-order diagrams with symbols as in Fig. 1.

The second-order contributions are illustrated in Fig. 2. Diagrams $A$ and $B$ yield vanishing contributions, while diagram $C$ can be written as a sum over lattice sites, by defining the quantities

$$
\begin{aligned}
& \varepsilon_{1}(\mathbf{R}, \tau):=\frac{1}{N_{s}} \sum_{\mathbf{k}} e^{i \mathbf{k} \cdot \mathbf{R}} e^{-\tau\left|\xi_{\mathbf{k}}\right|} \varepsilon_{\mathbf{k}} P_{\mathbf{k}}^{2}, \\
& \varepsilon_{2}(\mathbf{R}, \tau):=\frac{1}{N_{s}} \sum_{\mathbf{k}} e^{i \mathbf{k} \cdot \mathbf{R}} e^{-\tau\left|\xi_{\mathbf{k}}\right|} \varepsilon_{\mathbf{k}} Q_{\mathbf{k}}^{2} .
\end{aligned}
$$

We obtain

$$
\begin{aligned}
& \left\langle D(-\tau) H_{0} D(\tau)\right\rangle_{c} \\
& =2 N_{s} \sum_{\mathbf{R}} P(\mathbf{R}, 2 \tau) Q(\mathbf{R}, 2 \tau) \\
& \quad \times\left[\varepsilon_{2}(\mathbf{R}, 2 \tau) P(\mathbf{R}, 2 \tau)-\varepsilon_{1}(\mathbf{R}, 2 \tau) Q(\mathbf{R}, 2 \tau)\right] .
\end{aligned}
$$

The minimization of Eq. (14) with respect to $\eta$ yields the correlation energy

$$
\begin{aligned}
E_{\text {corr }} & :=\bar{E}-\langle H\rangle \\
& \approx-U^{2} \frac{\langle D D(\tau)\rangle_{c}^{2}}{\left\langle D(-\tau) H_{0} D(\tau)\right\rangle_{c}},
\end{aligned}
$$

which is readily evaluated numerically because the individual terms, Eqs. (24) and (27), are simple sums. The result has still to be minimized with respect to the parameter $\tau$. It turns out that $\tau$ depends weakly on $U$ (for small $U$ ), but more sensitively on the particle density $n$. Some results for the particular case of $U=1$ are given in Table I for a $1000 \times 1000$ square lattice. Both $\eta$ and $\tau$ are largest at half filling.

It is instructive to compare these results with the exact second-order term deduced by perturbation theory [44]. The

TABLE I. Variational parameters $\eta, \tau$ and correlation energy per site for $U=1, L=1000$, and various particle densities $n$, in comparison with the correlation energy $E_{\text {corr }}^{(\mathrm{ex})}$ obtained by secondorder perturbation theory $(U=1, L=1000)$.

\begin{tabular}{ccccc}
\hline \hline$n$ & $\eta$ & $\tau$ & $E_{\text {corr }} / L$ & $E_{\text {corr }}^{(\mathrm{ex})} / L$ \\
\hline 1.0 & 0.59633 & 0.19805 & -0.012169 & -0.012562 \\
0.9 & 0.57690 & 0.19286 & -0.011774 & -0.012072 \\
0.8 & 0.54594 & 0.18435 & -0.010787 & -0.010995 \\
0.7 & 0.51750 & 0.17618 & -0.009406 & -0.009553 \\
0.6 & 0.49427 & 0.16917 & -0.007774 & -0.007880 \\
0.5 & 0.47666 & 0.16349 & -0.006014 & -0.006092 \\
\hline \hline
\end{tabular}


TABLE II. Variational parameter $\eta^{(G)}$ and correlation energy $E_{\text {corr }}^{(G)}$ for the Gutzwiller ansatz, in comparison with the correlation energy $E_{\text {corr }}^{(\mathrm{ex})}$ obtained by second-order perturbation theory $(U=1$, $L=1000)$.

\begin{tabular}{lccc}
\hline \hline$n$ & $\eta^{(G)}$ & $E_{\text {corr }}^{(G)} / L$ & $E_{\text {corr }}^{(\mathrm{ex})} / L$ \\
\hline 1.0 & 0.14717 & -0.010220 & -0.012562 \\
0.9 & 0.14639 & -0.009961 & -0.012072 \\
0.8 & 0.14457 & -0.009236 & -0.010995 \\
0.7 & 0.14216 & -0.008139 & -0.009553 \\
0.6 & 0.13944 & -0.006771 & -0.007880 \\
0.5 & 0.13652 & -0.005246 & -0.006092 \\
\hline \hline
\end{tabular}

latter can be written as

$$
\begin{aligned}
E_{\mathrm{corr}}^{(\mathrm{ex})}= & \frac{U^{2}}{L^{2}} \sum_{\mathbf{k}_{1}, \mathbf{k}_{2}, \mathbf{k}_{3}, \mathbf{k}_{4}} \delta\left(\mathbf{k}_{1}-\mathbf{k}_{2}+\mathbf{k}_{3}-\mathbf{k}_{4}\right) \\
& \times \frac{P_{\mathbf{k}_{1}} Q_{\mathbf{k}_{2}} P_{\mathbf{k}_{3}} Q_{\mathbf{k}_{4}}}{\xi_{\mathbf{k}_{1}}-\xi_{\mathbf{k}_{2}}+\xi_{\mathbf{k}_{3}}-\xi_{\mathbf{k}_{4}}} \\
= & -N_{s} U^{2} \int_{0}^{\infty} d \tau \sum_{\mathbf{R}}[P(\mathbf{R}, \tau) Q(\mathbf{R}, \tau)]^{2} .
\end{aligned}
$$

The data shown in Table I confirm that our variational ansatz reproduces the exact second-order term to a high precision (97\% at half filling, 99\% for $n=0.5$ ). The corresponding results for the Gutzwiller ansatz, listed in Table II, are substantially less accurate $(81 \%$ of the exact correlation energy at half filling and $86 \%$ for $n=0.5$ ). This large improvement of the correlation energy by the parameter $\tau$ also holds for larger values of $U[23,24]$. We notice that the $\eta$ values for the variational ansatz including $\tau$ are much larger than those for the Gutzwiller ansatz (where $\tau=0$ ). This happens because with increasing $\tau$ the contribution of the region away from the Fermi surface is reduced and thus the cost in band energy due to the reduction of double occupancy is lowered, and $\eta$ assumes higher values than for $\tau=0$.

The relatively large values of the correlation parameter $\eta$ for small values of $U$ reflect the fact that small bare interactions do not necessarily imply weak correlations.

\section{SUPERCONDUCTING STATE}

\section{A. Reference state}

For superconductivity the reference state is the ground state of the mean-field Hamiltonian

$$
\begin{aligned}
H_{m}= & \sum_{\mathbf{k}}\left\{\xi_{\mathbf{k}}\left(c_{\mathbf{k} \uparrow}^{\dagger} c_{\mathbf{k} \uparrow}+c_{-\mathbf{k} \downarrow}^{\dagger} c_{-\mathbf{k} \downarrow}\right)\right. \\
& \left.-\Delta_{\mathbf{k}}\left(c_{\mathbf{k} \uparrow}^{\dagger} c_{-\mathbf{k} \downarrow}^{\dagger}+c_{-\mathbf{k} \downarrow} c_{\mathbf{k} \uparrow}\right)\right\},
\end{aligned}
$$

where $\xi_{\mathbf{k}}:=\varepsilon_{\mathbf{k}}-\mu$ and the gap parameter $\Delta_{\mathbf{k}}$ must have an appropriate symmetry, such as $d$ wave or $p$ wave. In this paper we restrict ourselves to $d$-wave symmetry, i.e.,

$$
\Delta_{\mathbf{k}}=\Delta_{0}\left(\cos k_{x}-\cos k_{y}\right) .
$$

The parameter $\mu$ is chosen in such a way that the average particle number is equal to a fixed value. For the correlated ground state (7) $\mu$ can be identified with the (true) chemical potential only for $\Delta_{\mathbf{k}}=0$ and $U=0$.

$H_{m}$ is diagonalized by the Bogoliubov transformation

$$
\begin{aligned}
c_{\mathbf{k} \uparrow} & =\cos \vartheta_{\mathbf{k}} \alpha_{\mathbf{k} \uparrow}+\sin \vartheta_{\mathbf{k}} \alpha_{-\mathbf{k} \downarrow}^{\dagger}, \\
c_{-\mathbf{k} \downarrow}^{\dagger} & =-\sin \vartheta_{\mathbf{k}} \alpha_{\mathbf{k} \uparrow}+\cos \vartheta_{\mathbf{k}} \alpha_{-\mathbf{k} \downarrow}^{\dagger},
\end{aligned}
$$

if $\vartheta_{\mathbf{k}}$ is chosen as

$$
\cos 2 \vartheta_{\mathbf{k}}=\frac{\xi_{\mathbf{k}}}{E_{\mathbf{k}}}, \quad \sin 2 \vartheta_{\mathbf{k}}=\frac{\Delta_{\mathbf{k}}}{E_{\mathbf{k}}}
$$

where

$$
E_{\mathbf{k}}=\sqrt{\xi_{\mathbf{k}}^{2}+\Delta_{\mathbf{k}}^{2}}
$$

is the excitation spectrum. The mean-field Hamiltonian now reads

$$
H_{m}=\sum_{\mathbf{k}}\left(\xi_{\mathbf{k}}-E_{\mathbf{k}}\right)+\sum_{\mathbf{k} \sigma} E_{\mathbf{k}} \alpha_{\mathbf{k} \sigma}^{\dagger} \alpha_{\mathbf{k} \sigma} .
$$

Its ground state $\left|\Psi_{m}\right\rangle$ is the vacuum for quasiparticles, $\alpha_{\mathbf{k} \sigma}\left|\Psi_{m}\right\rangle=0$. It is then easy to see that

$$
\begin{aligned}
c_{\mathbf{k} \sigma}(\tau)\left|\Psi_{m}\right\rangle & =e^{-\tau E_{k}} c_{\mathbf{k} \sigma}\left|\Psi_{m}\right\rangle, \\
c_{\mathbf{k} \sigma}^{\dagger}(\tau)\left|\Psi_{m}\right\rangle & =e^{-\tau E_{k}} c_{\mathbf{k} \sigma}^{\dagger}\left|\Psi_{m}\right\rangle .
\end{aligned}
$$

Three different functions appear in the Wick decomposition, the momentum distribution functions

$$
\begin{gathered}
P_{\mathbf{k}}:=\left\langle c_{\mathbf{k} \sigma}^{\dagger} c_{\mathbf{k} \sigma}\right\rangle=\frac{E_{\mathbf{k}}-\xi_{\mathbf{k}}}{2 E_{\mathbf{k}}}, \\
Q_{\mathbf{k}}:=\left\langle c_{\mathbf{k} \sigma} c_{\mathbf{k} \sigma}^{\dagger}\right\rangle=\frac{E_{\mathbf{k}}+\xi_{\mathbf{k}}}{2 E_{\mathbf{k}}},
\end{gathered}
$$

and the "Gor'kov function"

$$
F_{\mathbf{k}}:=\left\langle c_{-\mathbf{k} \downarrow} c_{\mathbf{k} \uparrow}\right\rangle=\left\langle c_{\mathbf{k} \uparrow}^{\dagger} c_{-\mathbf{k} \downarrow}^{\dagger}\right\rangle=\frac{\Delta_{\mathbf{k}}}{2 E_{\mathbf{k}}} .
$$

\section{B. Second-order expansion}

We are now prepared for carrying out explicitly the expansion (14) for a superconducting reference state. The contribution of zeroth order is given by

$$
\langle H\rangle=2 \sum_{\mathbf{k}} \varepsilon_{\mathbf{k}} P_{\mathbf{k}}+N_{s} U\left(\frac{n^{2}}{4}+f_{0}^{2}\right),
$$

where

$$
f_{0}:=\frac{1}{N_{s}} \sum_{\mathbf{k}} F_{\mathbf{k}} .
$$

For an order parameter with $p$ - or $d$-wave symmetry, the "average Gor'kov function" $f_{0}$ vanishes if both the lattice and the boundary conditions have fourfold rotational symmetry. In order to cope with slight deviations from this symmetry for finite system sizes (due to periodic-antiperiodic boundary conditions) we retain $f_{0}$ in the analytical expressions. For the large system sizes considered here the breaking of the fourfold rotational symmetry has very little effect. In recent variational Monte Carlo studies [45], with periodic-antiperiodic boundary conditions and $L$ up to 24 , striped phases have been found to be slightly more stable than homogeneous 
superconductivity. Because breaking of the fourfold rotational symmetry is expected to favor stripes and to weaken $d$-wave superconductivity, one may ask whether these results survive for larger system sizes or for symmetric boundary conditions.

The contribution of first order in $\eta$ has three terms

$$
\langle H D(\tau)\rangle_{c}=A+U(B+C),
$$

where $A$ comes from the hopping part of the Hamiltonian and $B, C$ from the interaction. They correspond to the three diagrams of Fig. 1, where a line can represent any of the three functions $P, Q, F$, and are given explicitly by

$$
\begin{aligned}
A= & 2 \sum_{\mathbf{k}} e^{-2 \tau E_{\mathbf{k}}} \varepsilon_{\mathbf{k}} F_{\mathbf{k}} S_{\mathbf{k}}, \\
B= & \sum_{\mathbf{k}} e^{-2 \tau E_{\mathbf{k}}} S_{\mathbf{k}}^{2}, \\
C= & \frac{1}{N_{s}^{2}} \sum_{\mathbf{p}, \mathbf{q}, \mathbf{l}, \mathbf{k}} e^{-\tau\left(E_{\mathbf{p}}+E_{\mathbf{q}}+E_{\mathbf{l}}+E_{\mathbf{k}}\right)} \delta(\mathbf{p}+\mathbf{q}+\mathbf{l}+\mathbf{k}) \\
& \times\left(P_{\mathbf{p}} Q_{\mathbf{q}}-F_{\mathbf{p}} F_{\mathbf{q}}\right)\left(P_{1} Q_{\mathbf{k}}-F_{\mathbf{l}} F_{\mathbf{k}}\right),
\end{aligned}
$$

where

$$
S_{\mathbf{k}}=n F_{\mathbf{k}}+f_{0} G_{\mathbf{k}}
$$

with

$$
G_{\mathbf{k}}=Q_{\mathbf{k}}-P_{\mathbf{k}}=\frac{\xi_{\mathbf{k}}}{E_{\mathbf{k}}}
$$

The triple summation in $C$ is replaced by a simple summation over lattice sites using Eq. (5) together with the Fourier transform

$$
P(\mathbf{R}, \tau):=\frac{1}{N_{s}} \sum_{\mathbf{k}} e^{i \mathbf{k} \cdot \mathbf{R}} e^{-\tau E_{\mathbf{k}}} P_{\mathbf{k}}
$$

and correspondingly for the other functions. We get

$$
C=N_{s} \sum_{\mathbf{R}}\left[P(\mathbf{R}, \tau) Q(\mathbf{R}, \tau)-F^{2}(\mathbf{R}, \tau)\right]^{2} .
$$

We now turn to the second-order contribution in Eq. (14). The diagrams are grouped according to the three general structures of Fig. 2 but, because of the various possibilities for lines (representing $P_{\mathbf{k}}, Q_{\mathbf{k}}$, or $F_{\mathbf{k}}$ ) and Hartree bubbles (density per spin or average Gor'kov function) there are many different specific diagrams, namely 29 of type $A, 18$ of type $B$, and 16 of type $C$. Nevertheless, the result can be presented in a relatively compact form, as shown in Appendix A. The numerical evaluation of the various terms requires only simple summations, either in $k$ or in $R$ space.

The second-order expansion of the particle number, Eq. (16), is effectuated in the same way. To deduce the corresponding formulas one simply has to replace $\varepsilon_{\mathbf{k}}$ by 1 and $U$ by 0 in the expression for the energy.

\section{Numerical procedure}

In the numerical calculations we have considered finite quadratic arrays of size $L \times L$ with $L$ up to 4000 . Thus the number of $\mathbf{k}$ points in the Brillouin zone is $N_{s}=L^{2}$. Periodicantiperiodic boundary conditions have been used, in order to reduce level degeneracies. For a given density $n=\bar{N} / N_{s}$
TABLE III. Gap parameters in the cases of full $\left(\tau_{\mathrm{opt}}\right)$ and "initial" optimization $\left(\tau_{0}\right)$, for $U=1, L=1000$, and three different densities $n$

\begin{tabular}{ccccc}
\hline \hline$n$ & $\tau_{0}$ & $\Delta_{0}\left(\tau_{0}\right)$ & $\tau_{\mathrm{opt}}$ & $\Delta_{0}\left(\tau_{\mathrm{opt}}\right)$ \\
\hline 1.0 & 0.198053 & 0.0004247 & 0.198882 & 0.0004252 \\
0.8 & 0.184349 & 0.0026698 & 0.186988 & 0.0027491 \\
0.6 & 0.169172 & 0.0020630 & 0.170660 & 0.0020878 \\
\hline \hline
\end{tabular}

and a given system size the particle number $\bar{N}$ is chosen in such a way that there be no ambiguity in the reference state at $\Delta_{0}=0$. For $n=0.8$ and $L=1000$ this is the case for $\bar{N}=800000$ because with this choice the "highest occupied molecular orbital" (HOMO) is completely full and the "lowest unoccupied molecular orbital" (LUMO) is completely empty and there is no degeneracy in the reference state. For a lattice of $100 \times 100$ sites a particle number of 8000 would not lead to a full-shell situation, the closest numbers satisfying this criterion are $\bar{N}=7996$ and $\bar{N}=8004$. For a finite gap parameter the constraint of a fixed particle number has to be satisfied very accurately, because the energy gained by pairing, the "condensation energy," is much smaller than the correlation energy. The results presented below have been obtained with a precision of at least $10^{-14}$ for the density $n$.

Four parameters have to be determined by minimizing the energy for a fixed density, namely $\tau, \eta, \Delta_{0}$, and $\mu$. To reduce the complexity of the problem, we use the fact that the parameters $\tau$ and $\Delta_{0}$ interfere weakly. Therefore we determine the optimal value of $\tau$ initially, i.e., for $\Delta_{0}=0$. We have checked that a full variational treatment of all parameters would only slightly increase the stability of the superconducting state. This is illustrated in Table III, where the full optimization is shown to enhance the gap parameter by about $1 \%$. Correspondingly, the condensation energy increases slightly. In what follows, we will restrict ourselves to the "initial optimization." Some examples of optimized parameters are given in Table IV for $0.6 \leqslant U \leqslant 1.2$. The parameter $\tau_{0}$ is practically $U$ independent in this range, while the correlation parameter $\eta$ is proportional to $U$ and the gap parameter $\Delta_{0}$ varies much more strongly. A more detailed discussion of the $U$ dependence of the gap parameter will be given in Sec. VI.

\section{ENERGETICS}

\section{A. Energy gain}

After the initial minimization with respect to $\tau$ for $\Delta_{0}=0$, the energy $\bar{E}\left(\eta, \mu, \Delta_{0}\right)$ is calculated for a fixed gap parameter $\Delta_{0}$ and minimized analytically with respect to $\eta$, while $\mu$ is

TABLE IV. Variational parameters $\tau_{0}, \eta, \Delta_{0}$ for $n=0.8, L=$ 1000 , and several values of $U$.

\begin{tabular}{cccc}
\hline \hline$U$ & $\tau_{0}$ & $\eta$ & $\Delta_{0}$ \\
\hline 0.6 & 0.184349 & 0.327026 & 0.0004204 \\
0.8 & 0.184349 & 0.436102 & 0.0010891 \\
1.0 & 0.184349 & 0.546204 & 0.0026698 \\
1.2 & 0.184349 & 0.660916 & 0.0067672 \\
\hline \hline
\end{tabular}




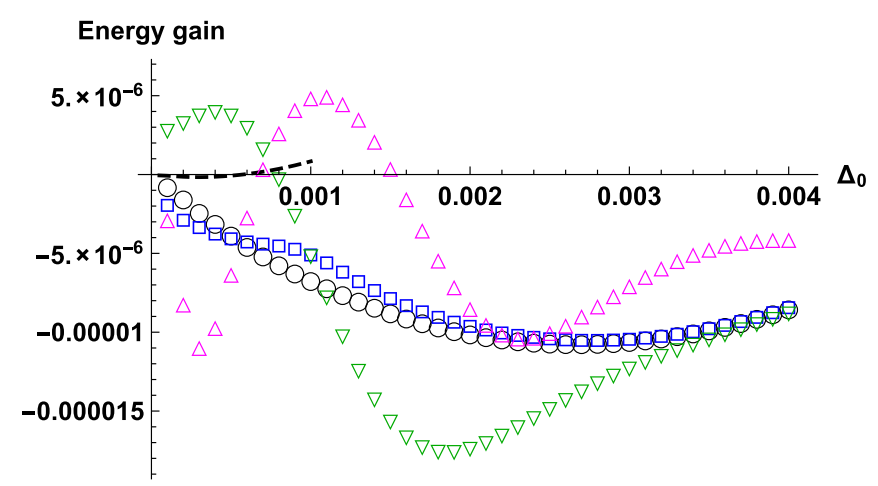

FIG. 3. Energy gain due to superconductivity as a function of the gap parameter $\Delta_{0}$ for $n=0.8$ and $U=1$. Symbols correspond to different system sizes, $L=1000$ (circles), $L=500$ (squares), $L=$ 200 (down-pointing triangles), and $L=100$ (up-pointing triangles). The dashed line represents results obtained with the Gutzwiller ansatz (for $L=1000$ ).

determined by the constraint of a fixed density. This yields the parameters $\eta\left(\Delta_{0}\right), \mu\left(\Delta_{0}\right)$, and the energy difference

$$
\varepsilon\left(\Delta_{0}\right):=\frac{1}{N_{s}}\left[\bar{E}\left(\Delta_{0}\right)-\bar{E}(0)\right] .
$$

Figure 3 shows this quantity for $U=1, n=0.8$, and four different system sizes. Negative values of $\varepsilon\left(\Delta_{0}\right)$ imply that the system is unstable with respect to superconductivity. Both for $L=100$ and for $L=200, \varepsilon\left(\Delta_{0}\right)$ exhibits two minima, but the two curves differ appreciably from each other and from curves for larger system sizes. By contrast, the results for $L=500$ and $L=1000$ are quite similar, with a single minimum at $\Delta_{0} \approx 0.0027$. These finite-size effects will be discussed in more detail in Sec. VIII.

For comparison, $\varepsilon\left(\Delta_{0}\right)$ is also presented in Fig. 3 for the Gutzwiller ansatz $(\tau=0)$. A minimum is again found, but its position is an order of magnitude below that obtained for finite $\tau$. Correspondingly, the energy gain is nearly two orders of magnitude smaller. Therefore the variational parameter $\tau$ enhances both the correlation energy (as shown in Sec. II) and the energy gain due to superconductivity. This is an important observation because one could imagine a poor correlation energy to be compensated by an artificially large condensation energy.

\section{B. Condensation energy}

The minimization of the energy yields the optimized values of the parameters $\Delta_{0}, \eta$ and $\mu$. The optimized gap parameter $\Delta_{0}$ [46] will be detailed in Sec. VI. Figure 4 shows the condensation energy, which we define as

$$
\varepsilon_{\mathrm{cond}}=\frac{1}{N_{s}}\left[\bar{E}(0)-\bar{E}_{\min }\left(\Delta_{0}\right)\right],
$$

as in BCS theory $[47,48]$. $\varepsilon_{\text {cond }}$ first increases when moving away from half filling (i.e., upon doping the parent half-filled system), passes through a maximum for $n \approx 0.8$ and then decreases. The values differ little when passing from $L=500$ to $L=2000$, except at half filling where $\varepsilon_{\text {cond }}$ decreases with increasing $L$. The calculations have been stopped at $n=0.5$.

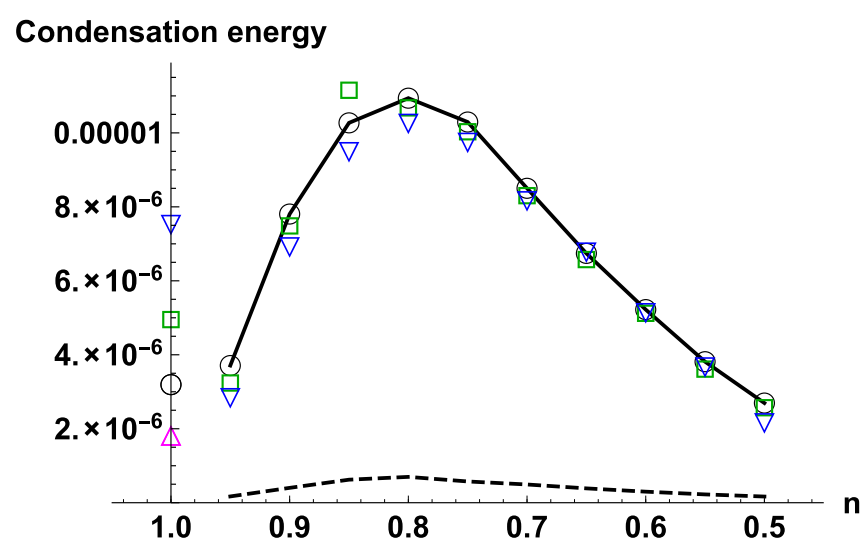

FIG. 4. Condensation energy for $U=1$ and different system sizes. Down-pointing triangles: $L=500$; squares: $L=1000$; circles and solid line: $L=2000$; up-pointing triangle: $L=4000$. The dashed line represents the BCS prediction, Eq. (49).

One expects that the condensation energy would decrease further for smaller densities, but in this case one would have to take into account the competing superconducting ground state with $p$-wave symmetry.

In BCS theory the condensation energy is related to the density of states $N(0)$ and the gap parameter $\Delta_{0}$ by the simple formula

$$
\varepsilon_{\text {cond }}^{(\mathrm{BCS})}=\frac{N(0)}{2} \Delta_{0}^{2} .
$$

Our results (solid line in Fig. 4) differ markedly from the BCS prediction (dashed line in Fig. 4). To find out whether the discrepancy can be attributed to the symmetry of the gap function ( $s$ wave due to a local attraction in BCS theory, $d$ wave in the present case), we have calculated the condensation energy for an extended Hubbard model with nearest-neighbor attraction using the mean-field ground state of Sec. IV A. Details are given in Appendix B. The results agree quite well with Eq. (49), which seems to hold approximately for any BCS-type theory. But why is the condensation energy so much larger in the present case than what would be predicted by BCS theory? We attribute the difference to the correlation energy, which involves not only the region very close to the Fermi energy, but also band states further away. In fact, the correlation parameter $\eta$-and therefore the correlation energy - increases with $\Delta_{0}$, as can be verified by comparing Tables I and IV (for $U=1$ ). The disparity would be even more pronounced if the fully optimized value of $\tau$ would be used for the superconducting state.

\section{Conventional or unconventional?}

Unconventional superconductivity is not a sharply defined concept. Sometimes it is associated with an unconventional symmetry of the order parameter, and sometimes the emphasis is on properties deviating markedly from BCS predictions or on the nonphonon glue mediating the effective attraction $[49,50]$. Superconductivity in the repulsive Hubbard model is unconventional in several respects, in its order parameter ( $d$ wave close to half filling, $p$ wave further away), in the mechanism (no phonons by assumption, maybe exchange of 


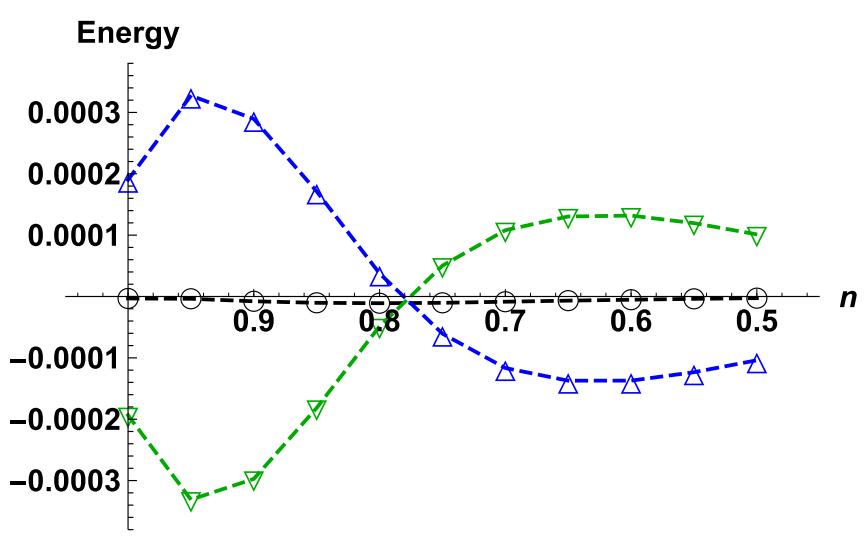

FIG. 5. Changes in kinetic (up-pointing triangles), potential (down-pointing triangles), and total energies (circles) for $U=1$ and $L=2000$.

spin fluctuations or no glue at all), and also in deviations from BCS predictions. Here we discuss the question of whether pairing is due to a decrease in potential energy, as in BCS theory, or rather due to an unconventional decrease in kinetic energy.

For the reduced BCS Hamiltonian it is easy to convince oneself that the kinetic energy cannot be lowered by pairing. For this model the normal state corresponds to the filled Fermi sea, which has the lowest possible kinetic energy for a given number of particles. Any interaction effect must then lead to an increase of kinetic energy, and superconductivity can only occur if this increase is overcompensated by a decrease in potential energy.

The issue of whether the condensation energy is generated by a gain in potential energy, as in BCS theory, or by a gain in kinetic energy has been addressed in the frameworks of spin-fluctuation exchange [51], cluster dynamical mean-field theory [52,53], and variational methods [32,35,36,54]. Quite generally, an unconventional gain in kinetic energy is found for large values of $U$ and/or weak doping, while a conventional gain in potential energy is obtained for heavy doping and/or not too large $U$, but the detailed predictions differ somewhat. For instance, different variational wave functions may give different answers for the same values of $U$ and for the same density $n$ [26].

It is straightforward to calculate individually the changes in potential and kinetic energies due to superconductivity within the present approach. The results, shown in Fig. 5 for $U=1$, are quite surprising, because the kinetic energy is lowered for heavy doping ( $n \lesssim 0.78$ ), while for weak doping $(n \gtrsim 0.78)$ there is a gain in potential energy, contrary to what is typically found in the numerical calculations mentioned above. There is no contradiction with the arguments given for the reduced BCS Hamiltonian, because the normal state $\left(\Delta_{0}=0\right)$ is correlated and has a kinetic energy exceeding its minimum value. Figure 5 also shows that the individual changes in potential and kinetic energies are much larger than the condensation energy. The same delicate balance between kinetic and potential energies has been observed some time ago on the basis of both variational Monte Carlo [55] and dynamic cluster calculations [53]. The corresponding results

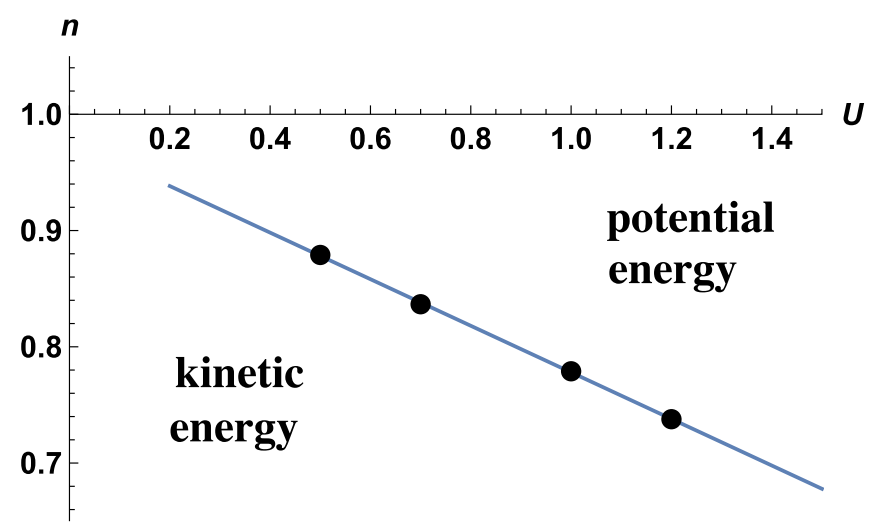

FIG. 6. Crossover from unconventional ("kinetic-energy lowering") to conventional ("potential-energy lowering") superconductivity for very small values of $U$. The numerical results (dots) have been calculated for $L_{x}=2000$ and are well converged. The line is a linear interpolation of the data points.

for the Gutzwiller ansatz will be presented in Sec. IX and are shown to be quite similar.

In order to scrutinize the unexpected kinetic-energy lowering, we have determined the crossing point between conventional and unconventional regimes for several values of $U$. The results shown in Fig. 6 indicate that for small values of $U$ kinetic-energy lowering is found for densities sufficiently far away from half filling. This is a region where umklapp scattering is expected to play a minor role, and we may speculate that this is the reason why electrons are "happy moving together" [56]. Figure 6 also shows that there is no real discrepancy with reports of conventional behavior for small values of $U$, because this conclusion is commonly reached on the basis of results obtained for $U \gtrsim 4[32,35,36,51-54,57]$.

\section{GAP PARAMETER}

The optimized gap parameter $\Delta_{0}$ is given in Fig. 7 for $U=1$ as a function of density for various system sizes. It shows a similar domelike shape as the condensation energy

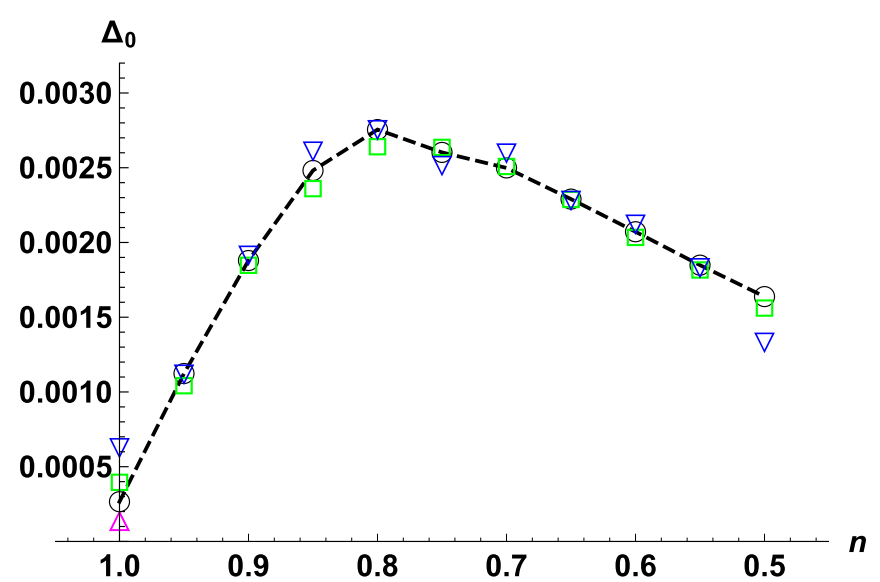

FIG. 7. Gap parameter as a function of electron density for $U=1$ and different system sizes. Down-pointing triangles: $L=500$; squares: $L=1000$; circles and dashed line: $L=2000$; up-pointing triangle: $L=4000$. 


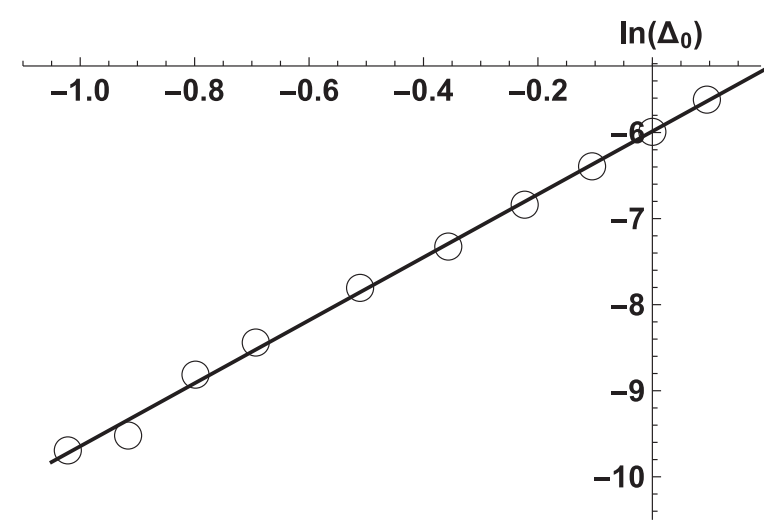

$\ln (U)$

FIG. 8. Gap parameter as a function of $U$ for $n=0.7$ and $L=$ 2000. The solid line is a linear fit through the data points.

(depicted in Fig. 4), although less pronounced. Again the results vary little with system size from $L=500$ to $L=2000$ except at half filling where they decrease steadily. Finite-size scaling (discussed in Sec. VIII) indicates that at half filling the gap parameter vanishes in the thermodynamic limit.

An interesting question is how $\Delta_{0}$ varies with $U$. Adopting the RPA expression for the effective interaction induced by the exchange of spin fluctuations $[58,59]$ in the small $U$ limit, one obtains an attraction proportional to $U^{2}$ and a BCS behavior for the gap parameter,

$$
\ln \frac{1}{\Delta_{0}} \propto \frac{1}{U^{2}} .
$$

The same dependence on $U$ has been found for the critical temperature (which is expected to be proportional to $\Delta_{0}$ ) using a renormalization-group approach [60].

To see whether the $U$ dependence of Eq. (50) also comes out from our variational method, we have calculated the gap parameter for various values of $U$. These are limited to a relatively small region because for too large values of $U$ the second-order expansion breaks down and for too small values of $U$ the tiny condensation energy would require a higher precision than what we used in the calculations. Figure 8 shows results for $n=0.7$ and $0.36 \leqslant U \leqslant 1$.1. A linear relationship between $\ln \Delta_{0}$ and $\ln U$ fits the data very well, giving

$$
\Delta_{0}=\left(U / U^{*}\right)^{\gamma}
$$

with $U^{*} \approx 5.2$ and $\gamma \approx 3.6$. By contrast, the functional behavior (50) does not fit these data. Results for $0.7<n<1$ are very similar, but because of the larger values of the correlation parameter $\eta$ (as compared to those for $n=0.7$ ) the range of $U$ values has to be reduced. It is interesting to note that $U^{*}$ is of the order of the interaction strength where a Mott transition would occur in the absence of antiferromagnetic ordering [61]. We notice that a power law has also been extracted from variational Monte Carlo calculations [35,55].

The apparent discrepancy between Eqs. (50) and (51) can have various causes. First, the values of $U$ used in the present calculations could still lie outside of the asymptotic region, and therefore a crossover to exponential behavior at still smaller values of $U$ cannot be excluded. Second, the "superconducting gap" has quite different meanings in different approaches. Thus in the renormalization-group approach of
Ref. [60] the gap is assumed to have the same asymptotic behavior as the characteristic energy scale below which the approach breaks down, while in the present approach $\Delta_{0}$ is a variational parameter. The two methods could yield quite different gap parameters, which could also be quite different from the location of the peak in the spectral density. Third, long-wavelength fluctuations have been neglected in the present approach. These could modify the $U$ dependence, in a similar way as already found for the antiferromagnetic order at half filling [62]. Fourth, the functional renormalizationgroup procedure cannot be worked out without approximations. The version used by Raghu and co-workers [60] to establish Eq. (50) works on a "one-loop" level and neglects both frequency-dependencies and self-energy insertions in the irreducible vertices [63]. These approximations are not innocent [64], but so far applications of more sophisticated "multiloop" approaches have been limited to temperaturedependent generalized susceptibilities [65]. Finally, we may wonder whether perturbative methods can yield the correct asymptotic behavior for $U \rightarrow 0$. We have already seen that the refinement of the Gutzwiller ansatz reduces dramatically the $U$ region where a low-order expansion in powers of the correlation parameter $\eta$ can be trusted. Further refinements towards the exact ground state could reduce further the convergence radius, which might vanish eventually.

\section{ORDER PARAMETER}

The physical interpretation of the gap parameter $\Delta_{0}$ is far from obvious for any variational treatment of a superconductor with strong correlations. Thus it cannot simply be associated with a pseudogap, although this may yield an appealing picture of weakly doped cuprates [66]. In fact, the value of $\Delta_{0}$ depends quite strongly on the choice of the wave function [26]. By contrast, the order parameter can be sharply defined as an expectation value, and it depends much less on details [67]. In "canonical" calculations (fixed particle number) the order parameter is deduced from the long-distance behavior of the pair-pair correlation function [68]. In the present "grand-canonical" approach it is defined as the pair amplitude on nearest-neighbor sites $\mathbf{R}, \mathbf{R}^{\prime}$,

$$
\Phi:=\frac{\left\langle\Psi\left|c_{\mathbf{R} \uparrow}^{\dagger} c_{\mathbf{R}^{\prime} \downarrow}^{\dagger}\right| \Psi\right\rangle}{\langle\Psi \mid \Psi\rangle}=\frac{1}{N_{s}} \sum_{\mathbf{k}} \cos k_{x} \frac{\left\langle\Psi\left|C_{\mathbf{k}}^{\dagger}\right| \Psi\right\rangle}{\langle\Psi \mid \Psi\rangle},
$$

where

$$
C_{\mathbf{k}}:=c_{\mathbf{k} \uparrow}^{\dagger} c_{-\mathbf{k} \downarrow}^{\dagger}
$$

creates a Cooper pair. The expansion in powers of $\eta$ proceeds in exactly the same way as for the hopping term and we find

$$
\begin{aligned}
\frac{\left\langle\Psi\left|C_{\mathbf{k}}^{\dagger}\right| \Psi\right\rangle}{\langle\Psi \mid \Psi\rangle}= & F_{\mathbf{k}}-\eta\left\langle\left(C_{\mathbf{k}}^{\dagger}+C_{\mathbf{k}}\right) D(\tau)\right\rangle_{c} \\
& +\frac{\eta^{2}}{2}\left[\left\langle\left(C_{\mathbf{k}}^{\dagger}+C_{\mathbf{k}}\right) D^{2}(\tau)\right\rangle_{c}\right. \\
& \left.+\left\langle D(-\tau)\left(C_{\mathbf{k}}^{\dagger}+C_{\mathbf{k}}\right) D(\tau)\right\rangle_{c}\right] .
\end{aligned}
$$

The zeroth-order term is just given by the Gor'kov function, as in BCS theory. The first-order term reads

$$
\left\langle\left(C_{\mathbf{k}}^{\dagger}+C_{\mathbf{k}}\right) D(\tau)\right\rangle_{c}=e^{-2 \tau E_{\mathbf{k}}} G_{\mathbf{k}}\left(n F_{\mathbf{k}}+f_{0} G_{\mathbf{k}}\right)
$$




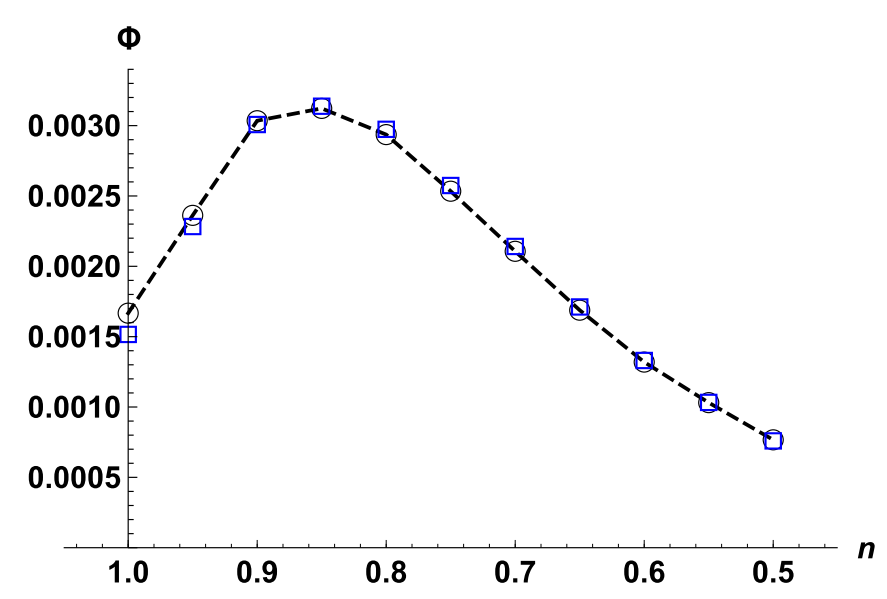

FIG. 9. Order parameter for $U=1$ and $L=1000$ as a function of the density $n$. Circles and the dashed line represent the secondorder expansion, squares the zeroth-order contribution.

and is represented by diagram $A$ of Fig. 1 . The second-order contributions correspond to the diagrams of Fig. 2 and are given explicitly in Appendix C. The numerical evaluation of the order parameter $\Phi$ is again straightforward, as only simple $k$ and $R$ sums have to be calculated. The result for $U=1$ and $L=1000$ is shown in Fig. 9. $\Phi$ has a maximum at $n \approx 0.85$ and is to a large extent given by the zeroth-order contribution (the Gor'kov function). For $n=1$ additional results for larger system sizes agree with the asymptotic behavior found for the gap parameter, which will be discussed below.

Figure 10 shows the $U$ dependence of the order parameter for $n=0.7$ and $L=2000$. The behavior is nearly indistinguishable from that of the gap parameter (Fig. 9) and again well described by the power law of Eq. (51), with $U^{*} \approx 6.7$ and $\gamma \approx 3.2$.

\section{FINITE-SIZE EFFECTS}

To highlight the size dependence of the gap parameter, we have plotted $\Delta_{0}$ vs $L^{-1}$ for three different densities in Fig. 11. Both for $n=0.85$ and for $n=0.6, \Delta_{0}$ shows a rather irregular behavior for $L \lesssim 300$, but then it approaches a finite limiting value for the largest system sizes. The wild

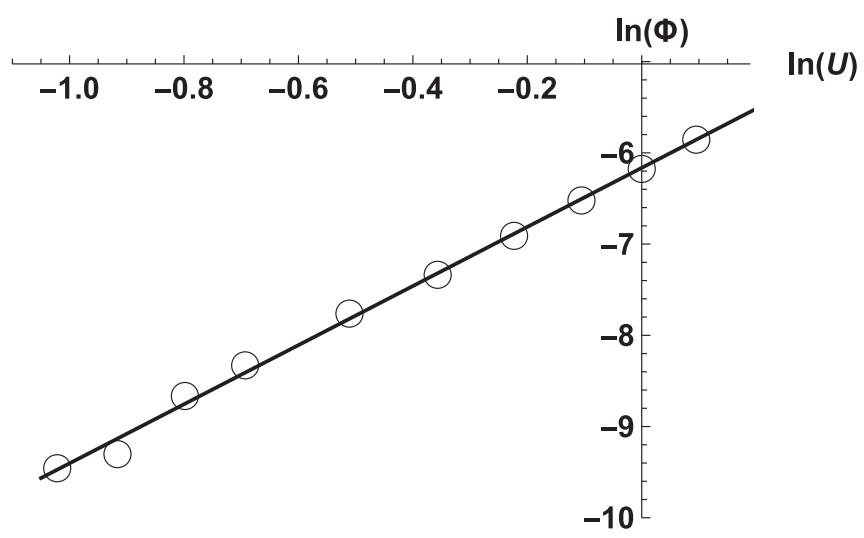

FIG. 10. Order parameter as a function of $U$ for $n=0.7$ and $L=$ 2000. The solid line is a linear fit through the data points.

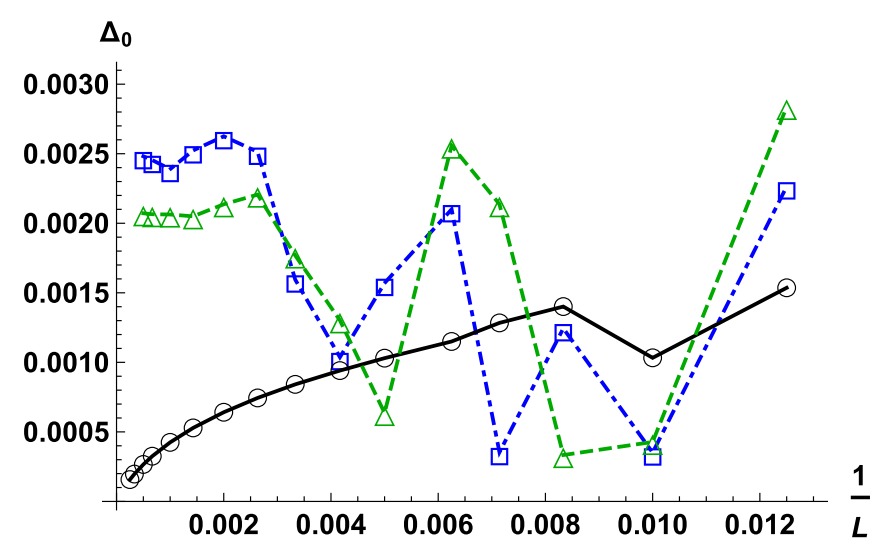

FIG. 11. Variation of the gap parameter with system size $(U=1)$. Circles and solid line: $n=1$; squares: $n=0.85$; triangles: $n=0.6$.

variation of $\Delta_{0}$ as a function of $L$ for small to intermediate system sizes originates most likely from irregular changes of the HOMO-LUMO gap $\Delta_{\mathrm{HL}}$ (the separation between the lowest unoccupied and the highest occupied single-particle levels $\varepsilon_{\mathbf{k}}$ ).

For $n=1$ the HOMO-LUMO gap is a smooth function,

$$
\Delta_{\mathrm{HL}}=4\left(1-\cos \frac{\pi}{L}\right) \approx \frac{2 \pi^{2}}{N_{s}} .
$$

Correspondingly, as one can see from Fig. 11, the superconducting gap $\Delta_{0}$ is also smooth as soon as the system size is large enough. The behavior for large $L$ is well described by the size dependence $\Delta_{0} \propto 1 / \sqrt{L}$, as evidenced in Fig. 12, and therefore $\Delta_{0}$ vanishes for $L \rightarrow \infty$. The figure also indicates that the boundary between regular and irregular behavior is defined by the equality of the two gaps, $\Delta_{0}=\Delta_{\mathrm{HL}}$.

It is quite remarkable that superconductivity fades away at half filling, because we did not include the possibility of a competing antiferromagnetic instability in the variational ansatz. Had we done so, antiferromagnetic long-range order would readily show up [69], in agreement with conventional

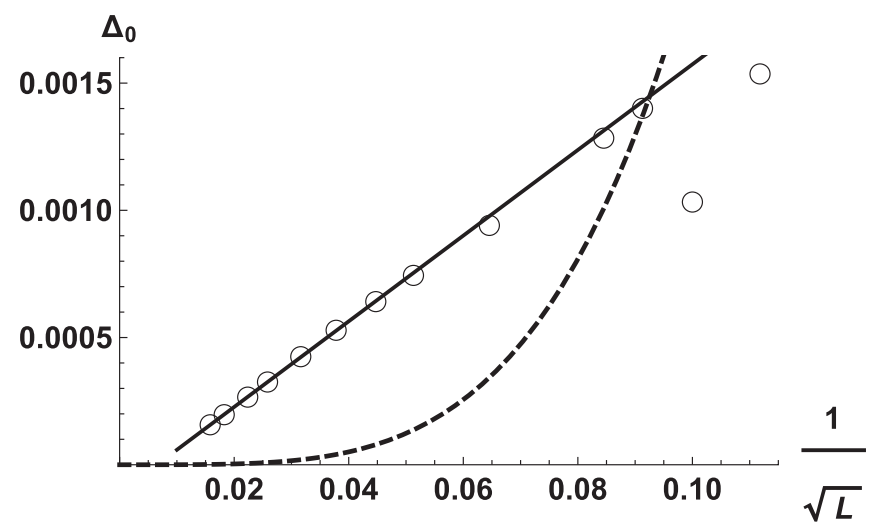

FIG. 12. Size dependence of the gap parameter for $U=1$ at half filling $(n=1)$. The dashed line corresponds to the HOMO-LUMO gap $\Delta_{\mathrm{HL}}$, given by Eq. (56). The solid line is a linear fit through those data points for which $\Delta_{0}$ exceeds $\Delta_{\mathrm{HL}}$. 


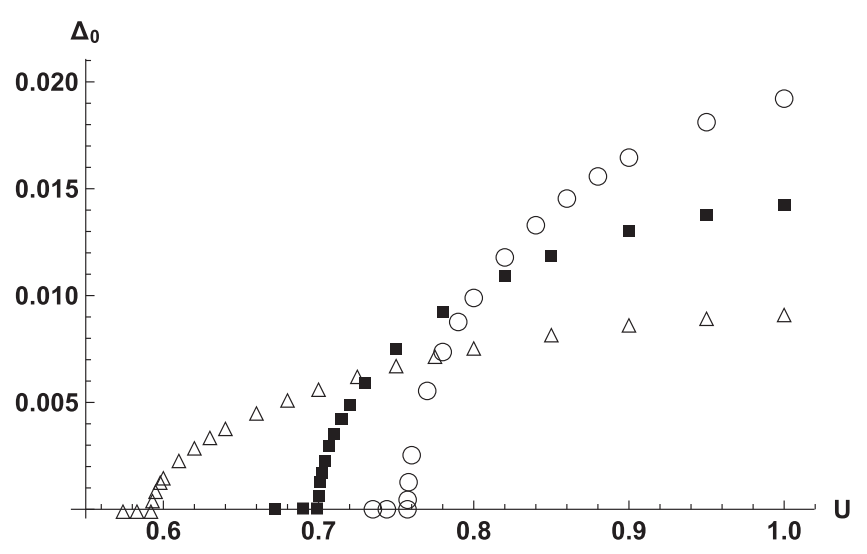

FIG. 13. $U$ dependence of the gap parameter for $n=0.85$ and small system sizes, $L=12$ (circles), $L=14$ (squares), and $L=20$ (triangles).

wisdom. The present results show that this competition is not necessary for quenching superconductivity at half filling.

Already in 1959 Anderson wondered what happens to a superconducting material if its size shrinks more and more [70]. He argued that superconductivity ceases as soon as the characteristic level spacing becomes larger than the energy gap of the bulk system. In the 1990s, Anderson's question was investigated thoroughly, both in spectroscopic experiments on ultrasmall aluminium particles and theoretically using the exact solution of the reduced BCS Hamiltonian [68]. Anderson's estimate was confirmed, at the same time the critical size was found to signal a crossover rather than a true transition.

We now address Anderson's question in the present context and search for the minimal length above which our wave function has superconducting order. Because of the irregularities described above (away from half filling) we do not vary $L$ for a fixed value of $U$, we rather vary $U$ for a fixed value of $L$. Therefore we determine the critical value of $U$, above which $\Delta_{0}$ is finite. Figure 13 shows the results for three small systems and a density of 0.85 . The gap parameter grows continuously from zero above a critical value $U_{c}(L)$. The larger $L$ the smaller $U_{c}(L)$. As to the numerical values of $U_{c}$, they are nearly an order of magnitude smaller than predicted by Anderson's criterion. Figure 14 shows similar re-

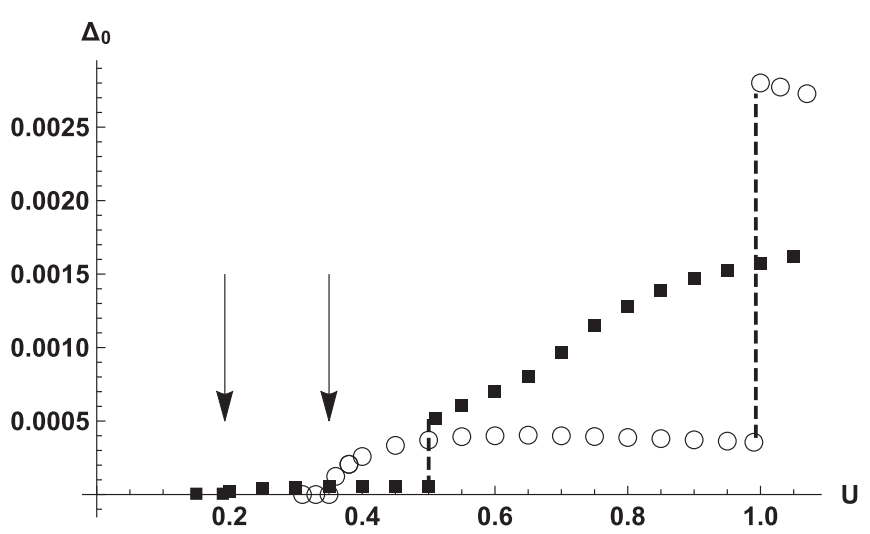

FIG. 14. $U$ dependence of the gap parameter for $n=0.85$ and intermediate system sizes, $L=100$ (circles) and $L=200$ (squares). The arrows indicate the critical points above which $\Delta_{0}$ is finite.

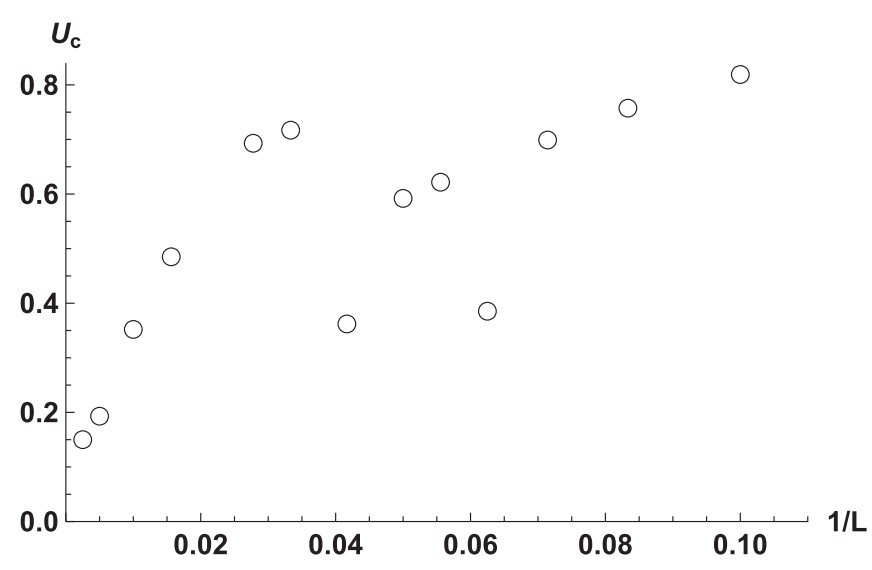

FIG. 15. Size dependence of the critical value $U_{c}$, below which superconducting order disappears, for $n=0.85$.

sults for systems of intermediate size, $L=100$ and 200. While $\Delta_{0}$ again increases first smoothly, there exist discontinuities, which result from energy curves with two minima, such as that shown in Fig. 3. On the left side of a jump the lower minimum has lower energy, on the right side the upper minimum is more stable. On average, the critical values $U_{c}$ decrease with system size, as exemplified in Fig. 15 for $n=0.85$. The data shown in the figure are consistent with a vanishing $U_{c}$ for $L \rightarrow \infty$, i.e., for an infinite system there is superconductivity for arbitrarily small $U$.

To explore the fluctuations of the gap parameter as a function of system size in a more quantitative way than above, we introduce coarse-graining for both gaps $\left(\Delta_{0}\right.$ and $\left.\Delta_{\mathrm{HL}}\right)$, by averaging over five neighboring (even) values of $L$. Results for $n=0.85$ and $U=1$, shown in Fig. 16, reveal that the fluctuations remain large above Anderson's critical size $(L \approx$ 100 in this case) and die out only when the superconducting gap spans several level spacings. Calculations for other values of $n$ and $U$ give similar results.

The overall evolution of superconducting order as a function of system size is thus governed by two characteristic sizes, a first one where order appears continuously, much

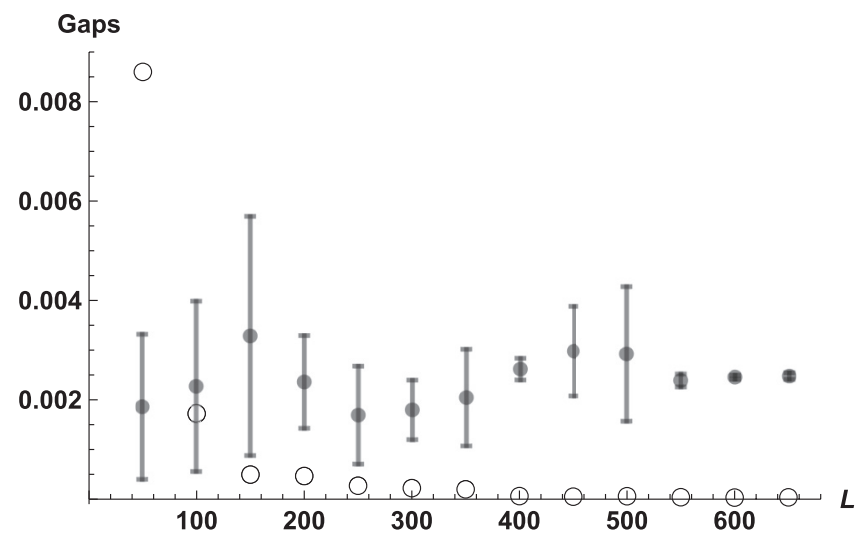

FIG. 16. Average gap parameter $\Delta_{0}$ (dots) and standard deviation (error bars) for $n=0.85, U=1$, and various (average) system sizes. The circles indicate the average HOMO-LUMO gap $\Delta_{\mathrm{HL}}$. Its standard deviation is of the order of the average value. 


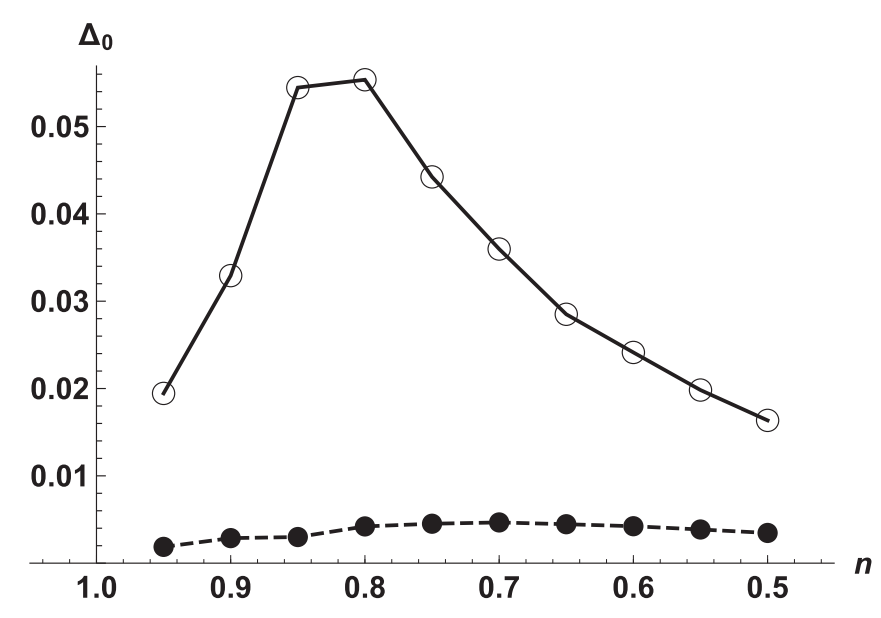

FIG. 17. Gap parameter as a function of electron density for $L=1000$, calculated with the Gutzwiller ansatz. Circles and solid line: $U=3$; dots and dashed line: $U=2$.

below Anderson's critical size, and a second one where the order parameter becomes well defined, far above Anderson's critical size. Similar finite-size effects should play a role in any numerical treatment of the two-dimensional Hubbard model on a finite lattice, be it quantum Monte Carlo, variational treatments, or dynamical mean-field theory. For $U$ of the order of the bandwidth $(U \approx 8)$ the typical size of the superconducting gap is found to be 0.1 [26]. This is also the typical size of the HOMO-LUMO gap for a $12 \times 12$ lattice. In this parameter regime fluctuations are expected to be substantial.

\section{GUTZWILLER ANSATZ}

It is worthwhile to compare the results obtained for the variational ansatz (7) with those deduced from the standard Gutzwiller wave function. The Gutzwiller predictions for energy and particle number can be found by putting $\tau=0$ in the corresponding expressions of Sec. IV and Appendix A. Because in this case the correlation parameter $\eta$ is much smaller than for the full ansatz, the expansion in powers of $\eta$ can be used for larger values of $U$.

Figure 17 shows the optimized gap value as a function of density for $U=2$ and $U=3$, where $\eta$ is of the order of 0.3 and 0.45 , respectively. The shape for $U=3$ is slightly more peaked than in the case of the full ansatz, Fig. 7, but the maximum occurs at about the same density. The gap values obtained for $U=2$ with the Gutzwiller ansatz are smaller than those of the full ansatz, which were calculated for $U=1$. This simply reflects the fact that superconductivity is strengthened by the additional term $e^{-\tau H_{m}}$ in Eq. (7).

Figure 18 shows the condensation energy together with the changes of kinetic and potential energy due to pairing for $U=$ 2. One notices a striking similarity to Fig. 5. There is again a change from a potential- to kinetic-energy driven pairing as the density decreases, although now the crossing occurs at a lower density. For $U=3$ the behavior is conventional, i.e., there is a gain in potential energy for all densities between 1 and 0.5 , accompanied by a loss in kinetic energy.

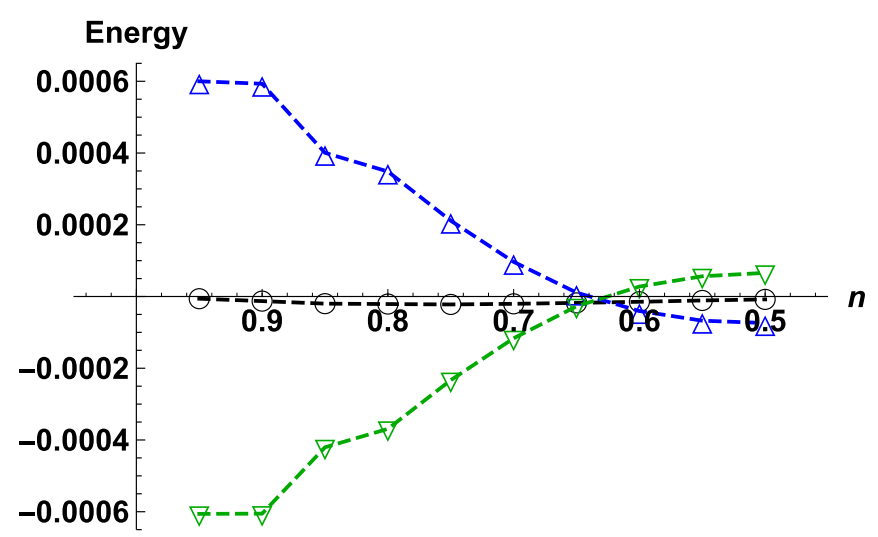

FIG. 18. Pairing-induced changes in kinetic (up-pointing triangles), potential (down-pointing triangles), and total energies (circles) for $U=2$ and $L=1000$, obtained with the Gutzwiller ansatz.

Finite-size effects for the Gutzwiller wave function are quite similar to those described for the full ansatz in Sec. VIII. At half filling, we find again $\Delta_{0} \propto 1 \sqrt{L}$ for large $L$. For other densities, there exists again a critical value $U_{c}$, below which $\Delta_{0}$ vanishes. For $U$ slightly above $U_{c}$ the behavior is like in Figs. 13 and 14. However, the values for the onset of superconductivity are appreciably higher, as shown in Table V. Irregular behavior is also detected for the conventional Gutzwiller ansatz, although maybe slightly less violent than in Fig. 11.

\section{SUMMARY AND OUTLOOK}

In this paper the two-dimensional repulsive Hubbard model has been scrutinized for $d$-wave superconductivity, using a refined Gutzwiller ansatz for the ground state. The operator $e^{-\eta D}$, which simply reduces double occupancy of a reference state (a BCS mean-field state in the present case), was supplemented by a term $e^{-\tau H_{m}}$, which involves the BCS mean-field Hamiltonian $H_{m}$. This ansatz is well suited for treating the small $U$ region. On the one hand, it admits a linked-cluster expansion in powers of the correlation parameter $\eta$, as in the case of the standard Gutzwiller ansatz. On the other hand, the energy is greatly improved by the additional term, as evidenced by a comparison with the expansion of the exact ground-state energy. Moreover, this approach has the advantage that large system sizes (millions of sites) can be treated to a very high precision. The drawback is that the method is limited to a relatively small interval of coupling

TABLE V. Critical values $U_{c}$ for $n=0.85$ and different system sizes. The values for the refined wave function (second column) are much smaller than those for the Gutzwiller ansatz (third column).

\begin{tabular}{lcc}
\hline \hline$L$ & $U_{c}(\tau>0)$ & $U_{c}(\tau=0)$ \\
\hline 12 & 0.757 & 1.89 \\
14 & 0.699 & 1.75 \\
20 & 0.592 & 1.45 \\
100 & 0.35 & 0.858 \\
200 & 0.193 & 0.449 \\
\hline \hline
\end{tabular}


strengths, $U_{1}<U<U_{2}$, where $U_{1} \approx 0.35$ (for lattices which are not larger than a few million sites) and $U_{2} \approx 1.2$ (to keep $\eta$ smaller than about 0.6).

The following main results were obtained:

(1) Superconductivity with $d$-wave symmetry exists away from half filling $(0.5 \leqslant n<1)$ with a maximum stability for $n \approx 0.8$.

(2) Both the gap parameter $\Delta_{0}$ and the order parameter $\Phi$ are an order of magnitude larger than in the case of the Gutzwiller ansatz.

(3) Correspondingly, the condensation energy also has a maximum for $n \approx 0.8$ and is larger by nearly two orders of magnitude than in the Gutzwiller ansatz.

(4) The pairing is due to a lowering of the kinetic energy for small enough $U$ and for densities not too close to half filling.

(5) For a given lattice size a minimum value $U_{c}$ is required for superconductivity to emerge. This value decreases steadily with increasing size. We conclude that for an infinite system superconductivity exists for arbitrarily small $U$.

(6) Our findings resolve a discrepancy between variational Monte Carlo studies, which did not find signatures of superconductivity below some critical value of $U$ (of the order of 4) and perturbative treatments such as the functional renormalization group or the fluctuation-exchange approximation, which do find a superconducting instability for small values of $U$. The main reason for the failure of variational Monte Carlo calculations is that the system sizes that can be treated are not large enough for the small gap parameters found for $U \approx 1$.

(7) In the special case of a half-filled band $(n=1) \Delta_{0}$ and $\Phi$ vanish as the system size tends to infinity.

Four variational parameters have been inserted into the trial wave function, the gap parameter $\Delta_{0}$, the "chemical potential" $\mu$, the correlation parameter $\eta$, and $\tau$, the inverse of a soft energy cutoff. The parameter $\tau$ can also be interpreted as a characteristic imaginary time. The exact second-order contribution, Eq. (29), is indeed an integral over the imaginary time $\tau$. The corresponding variational term, Eq. (24), looks very similar, but the integral is replaced by the integrand at "time" $\tau$. Since this characteristic time strengthens superconductivity, one may wonder whether its role is to introduce retardation in some effective interaction. It would be worthwhile to study this question thoroughly.

Other questions could also be addressed, such as $p$-wave superconductivity (which is expected to dominate for small densities $n$ ), the competition between superconductivity and antiferromagnetism (which is expected to be weak in view of the vanishing superconducting order parameter at half filling, where antiferromagnetic ordering is strongest), or the effect of next-nearest-neighbor hopping $t^{\prime}$ (which could bring back superconductivity at half filling).

\section{ACKNOWLEDGMENTS}

This work has been supported by the Swiss National Science Foundation Grant No. 200020-132698. I have profited from a close collaboration with M. Menteshashvili at an early stage of this work and also from useful discussions with F. Gebhard and J. Bünemann. I also thank H. Yokoyama and M. Ogata for having provided their variational Monte Carlo data.

\section{APPENDIX A: SECOND-ORDER TERMS FOR THE ENERGY}

The second-order contributions of Eq. (14) can be grouped according to the diagrams of Fig. 2, and we write

$$
\begin{aligned}
\left\langle H_{0} D^{2}(\tau)\right\rangle_{c} & =A_{1}+B_{1}+C_{1}, \\
\left\langle D(-\tau) H_{0} D(\tau)\right\rangle_{c} & =A_{2}+B_{2}+C_{2} .
\end{aligned}
$$

Proceeding as in Sec. III, we introduce the quantities

$$
\varepsilon_{v}(\mathbf{R}, 2 \tau)=\frac{1}{N_{s}} \sum_{\mathbf{k}} e^{i \mathbf{k} \cdot \mathbf{R}} \varepsilon_{\mathbf{k}} e^{-2 \tau E_{\mathbf{k}}} g_{\mathbf{k} v},
$$

where

$$
g_{\mathbf{k} v}=\left\{\begin{array}{ll}
P_{\mathbf{k}}^{2}, & v=1 \\
Q_{\mathbf{k}}^{2}, & v=2 \\
F_{\mathbf{k}}^{2}, & v=3 \\
F_{\mathbf{k}} G_{\mathbf{k}}, & v=4
\end{array} .\right.
$$

All three diagrams of Fig. 2 contribute if the gap parameter is finite and we write, correspondingly,

$$
\begin{aligned}
\left\langle H_{0} D^{2}(\tau)\right\rangle_{c} & =A_{1}+B_{1}+C_{1}, \\
\left\langle D(-\tau) H_{0} D(\tau)\right\rangle_{c} & =A_{2}+B_{2}+C_{2} .
\end{aligned}
$$

We find

$$
\begin{aligned}
A_{1}= & 2 \sum_{\mathbf{k}} e^{-2 \tau E_{\mathbf{k}}} \varepsilon_{\mathbf{k}} F_{\mathbf{k}} S_{\mathbf{k}}\left(n G_{\mathbf{k}}-4 f_{0} F_{\mathbf{k}}\right), \\
A_{2}= & 2 \sum_{\mathbf{k}} e^{-4 \tau E_{\mathbf{k}}} \varepsilon_{\mathbf{k}} G_{\mathbf{k}} S_{\mathbf{k}}^{2}, \\
B_{1}= & \frac{2}{N_{s}} \sum_{\mathbf{k}, \mathbf{q}} e^{-2 \tau E_{\mathbf{k}}} \varepsilon_{\mathbf{k}} F_{\mathbf{k}} S_{\mathbf{q}}\left(G_{\mathbf{k}} G_{\mathbf{q}}+4 F_{\mathbf{k}} F_{\mathbf{q}}\right), \\
B_{2}= & \frac{4}{N_{s}} \sum_{\mathbf{k}, \mathbf{q}} e^{-2 \tau\left(E_{\mathbf{k}}+2 E_{\mathbf{q}}\right)} \varepsilon_{\mathbf{k}} F_{\mathbf{k}} S_{\mathbf{q}} \\
& \times\left(-Q_{\mathbf{k}} P_{\mathbf{q}}-P_{\mathbf{k}} Q_{\mathbf{q}}+2 F_{\mathbf{k}} F_{\mathbf{q}}\right), \\
C_{1}= & 4 N_{s} \sum_{\mathbf{R}}\left[P(\mathbf{R}) Q(\mathbf{R})-F^{2}(\mathbf{R})\right] \\
& \times\left[\varepsilon_{3}(\mathbf{R}, 2 \tau) G(\mathbf{R})-\varepsilon_{4}(\mathbf{R}, 2 \tau) F(\mathbf{R})\right], \\
C_{2}= & 2 N_{s} \sum_{\mathbf{R}}\left[P(\mathbf{R}, 2 \tau) Q(\mathbf{R}, 2 \tau)-F^{2}(\mathbf{R}, 2 \tau)\right] \\
& \times\left[\varepsilon_{2}(\mathbf{R}, 2 \tau) P(\mathbf{R}, 2 \tau)-\varepsilon_{1}(\mathbf{R}, 2 \tau) Q(\mathbf{R}, 2 \tau)\right. \\
& \left.+\varepsilon_{3}(\mathbf{R}, 2 \tau) G(\mathbf{R}, 2 \tau)-2 \varepsilon_{4}(\mathbf{R}, 2 \tau) F(\mathbf{R}, 2 \tau)\right],
\end{aligned}
$$

where $S_{\mathbf{k}}$ and $G_{\mathbf{k}}$ are given by Eqs. (43) and (44), respectively, and $P(\mathbf{R}):=P(\mathbf{R}, 0)$, and so on. $A_{1}, A_{2}, C_{1}$, and $C_{2}$ are simple sums, but also in $B_{1}$ and $B_{2}$ there are no true double sums because the $\mathbf{k}$ - and $\mathbf{q}$-dependent terms can be handled independently.

For periodic boundary conditions, where $\varepsilon_{\mathbf{k}}$ is even under a reflection by the diagonal and $\Delta_{\mathbf{k}}$ is odd, the above expressions are simplified. We have chosen periodic-antiperiodic boundary conditions, for which this symmetry does not hold as long as $L$ remains finite. Therefore we have used the full expressions (A5) in the computations. 


\section{APPENDIX B: BCS PAIRING FOR NEAREST-NEIGHBOR ATTRACTION}

The extended Hubbard model with repulsive on-site and attractive nearest-neighbor interactions, defined by the Hamiltonian

$$
H=\sum_{\mathbf{k} \sigma} \varepsilon_{\mathbf{k}} c_{\mathbf{k} \sigma}^{\dagger} c_{\mathbf{k} \sigma}+U \sum_{\mathbf{R}} n_{\mathbf{R} \uparrow} n_{\mathbf{R} \downarrow}-V \sum_{\left\langle\mathbf{R}, \mathbf{R}^{\prime}\right\rangle} n_{\mathbf{R}} n_{\mathbf{R}^{\prime}}
$$

$\left(U>0, V>0, n_{\mathbf{R}}:=\sum_{\sigma} n_{\mathbf{R} \sigma}\right)$, is unstable with respect to $d$-wave pairing at the BCS mean-field level. Using the ansatz of Sec. IV A, one readily finds the expectation value

$$
\begin{aligned}
\frac{1}{N_{s}}\langle H\rangle= & \frac{2}{N_{s}} \sum_{\mathbf{k}} \varepsilon_{\mathbf{k}} P_{\mathbf{k}}+U\left(\frac{n^{2}}{4}+f_{0}^{2}\right) \\
& -2 V\left\{n^{2}-\sum_{\alpha=x, y}\left[\left(\frac{1}{N_{s}} \sum_{\mathbf{k}} \cos k_{\alpha} P_{\mathbf{k}}\right)^{2}\right.\right. \\
& \left.\left.-\left(\frac{1}{N_{s}} \sum_{\mathbf{k}} \cos k_{\alpha} F_{\mathbf{k}}\right)^{2}\right]\right\},
\end{aligned}
$$

where $P_{\mathbf{k}}, F_{\mathbf{k}}$, and $f_{0}$ are defined, respectively, by Eqs. (37), (38), and (40).
TABLE VI. Gap parameter $\Delta_{0}$ and condensation energy $\varepsilon_{c}$ for different densities $n$ and interaction strengths $V$, as obtained by minimizing the energy (B2) for $L=1000$. To get the ratio $\varepsilon_{c} /\left[N(0) \Delta_{0}^{2}\right]$, one also needs the density of states at the Fermi energy, $N(0)$. We find $N(0) \approx 0.18399$ for $n=0.8$ and $N(0) \approx 0.13912$ for $n=0.6$ in the thermodynamic limit, $L \rightarrow \infty$.

\begin{tabular}{lcccc}
\hline \hline$n$ & $V$ & $\Delta_{0}$ & $\varepsilon_{c}$ & $\varepsilon_{c} /\left[N(0) \Delta_{0}^{2}\right]$ \\
\hline 0.8 & 0.5 & 0.010131 & $1.541 \times 10^{-5}$ & 0.8159 \\
0.8 & 0.8 & 0.089255 & $1.187 \times 10^{-3}$ & 0.8096 \\
0.8 & 1.0 & 0.176589 & $4.555 \times 10^{-3}$ & 0.7939 \\
0.6 & 0.5 & 0.000099 & $5.936 \times 10^{-10}$ & 0.4376 \\
0.6 & 0.8 & 0.009960 & $6.738 \times 10^{-6}$ & 0.4881 \\
0.6 & 1.0 & 0.047185 & $1.480 \times 10^{-4}$ & 0.4777 \\
\hline \hline
\end{tabular}

The minimization of this expression with respect to the gap parameter $\Delta_{0}$ for a fixed density $n$ (this constraint determines the chemical potential $\mu$ ) gives equilibrium values for $\Delta_{0}$ and for the condensation energy $\varepsilon_{c}$, as shown in Table VI. Together with the density of states $N(0)$ these data allow us to find the combination $\varepsilon_{c} /\left[N(0) \Delta_{0}^{2}\right]$, which is just $\frac{1}{2}$ in the original BCS theory for $s$-wave pairing. The corresponding numbers of Table VI for $d$-wave pairing are all quite close to $\frac{1}{2}$ although gap parameters and condensation energies vary by several orders of magnitude.

\section{APPENDIX C: SECOND-ORDER TERMS FOR THE ORDER PARAMETER}

The second-order terms of the expansion (54) correspond to the diagrams of Fig. 2, and we write

$$
\begin{aligned}
\left\langle\left(C_{\mathbf{k}}^{\dagger}+C_{\mathbf{k}}\right) D^{2}(\tau)\right\rangle_{c} & =A_{1}+B_{1}+C_{1}, \\
\left\langle D(-h)\left(C_{\mathbf{k}}^{\dagger}+C_{\mathbf{k}}\right) D(\tau)\right\rangle_{c} & =A_{2}+B_{2}+C_{2} .
\end{aligned}
$$

Using the notations of Secs. IV A and IV B, we find

$$
\begin{aligned}
A_{1} & =e^{-2 \tau E_{\mathbf{k}}} G_{\mathbf{k}} S_{\mathbf{k}}\left(n G_{\mathbf{k}}-4 f_{0} F_{\mathbf{k}}\right), \\
B_{1} & =e^{-2 \tau E_{\mathbf{k}}} G_{\mathbf{k}} \frac{1}{N_{s}} \sum_{\mathbf{q}} S_{\mathbf{q}}\left(G_{\mathbf{k}} G_{\mathbf{q}}+4 F_{\mathbf{k}} F_{\mathbf{q}}\right), \\
C_{1} & =2 e^{-2 \tau E_{\mathbf{k}}} G_{\mathbf{k}} \sum_{\mathbf{R}} \cos \mathbf{k} \cdot \mathbf{R}\left[F^{2}(\mathbf{R})-P(\mathbf{R}) Q(\mathbf{R})\right]\left[G_{\mathbf{k}} F(\mathbf{R})-F_{\mathbf{k}} G(\mathbf{R})\right],
\end{aligned}
$$

and

$$
\begin{aligned}
& A_{2}=-4 e^{-2 \tau E_{\mathbf{k}}} F_{\mathbf{k}} S_{\mathbf{k}}^{2}, \\
& B_{2}=e^{-4 \tau E_{\mathbf{k}}} G_{\mathbf{k}} \frac{2}{N_{s}} \sum_{\mathbf{q}} e^{-4 \tau E_{\mathbf{q}}} S_{\mathbf{q}}\left(-Q_{\mathbf{k}} P_{\mathbf{q}}-P_{\mathbf{k}} Q_{\mathbf{q}}+2 F_{\mathbf{k}} F_{\mathbf{q}}\right), \\
& C_{2}=4 e^{-2 \tau E_{\mathbf{k}}} F_{\mathbf{k}} \sum_{\mathbf{R}} \cos \mathbf{k} \cdot \mathbf{R}\left[F^{2}(\mathbf{R}, 2 \tau)-P(\mathbf{R}, 2 \tau) Q(\mathbf{R}, 2 \tau)\right]\left[P_{\mathbf{k}} Q(\mathbf{R}, 2 \tau)+Q_{\mathbf{k}} P(\mathbf{R}, 2 \tau)-2 F_{\mathbf{k}} F(\mathbf{R}, 2 \tau)\right] .
\end{aligned}
$$

[1] P. W. Anderson, New approach to the theory of superexchange interactions, Phys. Rev. 115, 2 (1959).

[2] M. C. Gutzwiller, Effect of Correlation on the Ferromagnetism of Transition Metals, Phys. Rev. Lett. 10, 159 (1963).
[3] J. Hubbard, Electron correlations in narrow energy bands, Proc. R. Soc. London, Ser. A 276, 238 (1963).

[4] J. Kanamori, Electron correlation and ferromagnetism of transition metals, Prog. Theor. Phys. 30, 275 (1963). 
[5] W. F. Brinkman and T. M. Rice, Application of Gutzwiller's variational method to the metal-insulator transition, Phys. Rev. B 2, 4302 (1970).

[6] D. Vollhardt, Normal ${ }^{3} \mathrm{He}$, an almost localized Fermi liquid, Rev. Mod. Phys. 56, 99 (1984).

[7] P. W. Anderson, The resonating valence bond state in $\mathrm{La}_{2} \mathrm{CuO}_{4}$, Science 235, 1196 (1987).

[8] W. Kohn and J. M. Luttinger, New Mechanism for Superconductivity, Phys. Rev. Lett. 15, 524 (1965).

[9] P. A. Lee, N. Nagaosa, and X. G. Wen, Doping a Mott insulator: Physics of high-temperature superconductivity, Rev. Mod. Phys. 78, 17 (2006).

[10] D. J. Scalapino, A common thread: The pairing interaction for unconventional superconductors, Rev. Mod. Phys. 84, 1383 (2012).

[11] B. Edegger, V. N. Muthukumar, and C. Gros, Gutzwiller-RVB theory of high-temperature superconductivity: Results from renormalized mean-field theory and variational Monte Carlo calculations, Adv. Phys. 56, 927 (2007).

[12] M. Ogata and H. Fukuyama, The $t-J$ model for the oxide high$T_{c}$ superconductors, Rep. Prog. Phys. 71, 036501 (2008).

[13] T. Maier, M. Jarrell, T. Pruschke, and M. H. Hettler, Quantum cluster theories, Rev. Mod. Phys. 77, 1027 (2005).

[14] G. Rohringer, H. Hafermann, A. Toschi, A. A. Katanin, A. E. Antipov, M. I. Katsnelson, A. I. Lichtenstein, A. N. Rubtsov, and K. Held, Diagrammatic routes to nonlocal correlations beyond dynamical mean field theory, Rev. Mod. Phys. 90, 025003 (2018).

[15] W. Metzner, M. Salmhofer, C. Honerkamp, V. Meden, and K. Schönhammer, Functional renormalization group approach to correlated fermion systems, Rev. Mod. Phys. 84, 299 (2012).

[16] D. Zanchi and H. J. Schulz, Weakly correlated electrons on a square lattice: Renormalization-group theory, Phys. Rev. B 61, 13609 (2000).

[17] C. J. Halboth and W. Metzner, Renormalization-group analysis of the two-dimensional Hubbard model, Phys. Rev. B 61, 7364 (2000).

[18] C. Honerkamp, M. Salmhofer, N. Furukawa, and T. M. Rice, Breakdown of the Landau Fermi liquid in two dimensions due to umklapp scattering, Phys. Rev. B 63, 035109 (2001).

[19] B. Binz, D. Baeriswyl, and B. Douçot, Weakly interacting electrons and the renormalization group, Ann. Phys. (Leipzig) 12, 704 (2003).

[20] S. Friederich, H. C. Krahl, and C. Wetterich, Functional renormalization for spontaneous symmetry breaking in the Hubbard model, Phys. Rev. B 83, 155125 (2011).

[21] A. Eberlein and W. Metzner, Superconductivity in the twodimensional $t$ - $t^{\prime}$ Hubbard model, Phys. Rev. B 89, 035126 (2014).

[22] D. Baeriswyl, Variational schemes for many-electron systems, Springer Ser. Solid-State Sci. 69, 183 (1987).

[23] H. Otsuka, Variational Monte Carlo studies of the Hubbard model in one and two dimensions, J. Phys. Soc. Jpn. 61, 1645 (1992).

[24] M. Dzierzawa, D. Baeriswyl, and M. Di Stasio, Variational wave functions for the Mott transition: The $1 / r$ Hubbard chain, Phys. Rev. B 51, 1993(R) (1995).

[25] D. Tahara and M. Imada, Variational Monte Carlo method combined with quantum-number projection and multi-variable optimization, J. Phys. Soc. Jpn. 77, 114701 (2008).
[26] D. Eichenberger and D. Baeriswyl, Superconductivity and antiferromagnetism in the two-dimensional Hubbard model: A variational study, Phys. Rev. B 76, 180504(R) (2007).

[27] T. Yanagisawa, Crossover from weakly to strongly correlated regions in the two-dimensional Hubbard model, J. Phys. Soc. Jpn. 85, 114707 (2016).

[28] T. Yanagisawa, Antiferromagnetism, superconductivity and phase diagram in the two-dimensional Hubbard model: Offdiagonal wave function Monte Carlo studies, J. Phys. Soc. Jpn. 88, 054702 (2019).

[29] R. Hlubina, Phase diagram of the weak-coupling twodimensional $t-t^{\prime}$ Hubbard model at low and intermediate electron density, Phys. Rev. B 59, 9600 (1999).

[30] F. Šimkovic, X. W. Liu, Y. Deng, and E. Kozik, Ground-state phase diagram of the repulsive fermionic $t-t^{\prime}$ Hubbard model on the square lattice from weak coupling, Phys. Rev. B 94, 085106 (2016).

[31] T. Giamarchi and C. Lhuillier, Phase diagrams of the twodimensional Hubbard and $t-J$ models by a variational Monte Carlo method, Phys. Rev. B 43, 12943 (1991).

[32] J. Kaczmarczyk, J. Spalek, T. Schickling, and J. Bünemann, Superconductivity in the two-dimensional Hubbard model: Gutzwiller wave function solution, Phys. Rev. B 88, 115127 (2013).

[33] W. Metzner and D. Vollhardt, Ground-State Properties of Correlated Fermions: Exact Analytic Results for the Gutzwiller Wave Function, Phys. Rev. Lett. 59, 121 (1987).

[34] T. A. Kaplan, P. Horsch, and P. Fulde, Close Relation between Localized-Electron Magnetism and the Paramagnetic Wave Function of Completely Itinerant Electrons, Phys. Rev. Lett. 49, 889 (1982).

[35] H. Yokoyama, Y. Tanaka, M. Ogata, and H. Tsuchiura, Crossover of superconducting properties and kinetic-energy gain in two-dimensional Hubbard model, J. Phys. Soc. Jpn. 73, 1119 (2004).

[36] H. Yokoyama, M. Ogata, Y. Tanaka, K. Kobayashi, and H. Tsuchiura, Crossover between BCS superconductor and doped Mott insulator of $d$-wave pairing state in twodimensional Hubbard model, J. Phys. Soc. Jpn. 82, 014707 (2013).

[37] H. Yokoyama and H. H. Shiba, Variational Monte-Carlo studies of the Hubbard model: Intersite correlation effects, J. Phys. Soc. Jpn. 59, 3669 (1990).

[38] M. Capello, F. Becca, M. Fabrizio, S. Sorella, and E. Tosatti, Variational Description of Mott Insulators, Phys. Rev. Lett. 94, 026406 (2005).

[39] R. Kaneko, L. F. Tocchio, R. Valenti, F. Becca, and C. Gros, Spontaneous symmetry breaking in correlated wave functions, Phys. Rev. B 93, 125127 (2016).

[40] L. F. Tocchio, F. Becca, A. Parola, and S. Sorella, Role of backflow correlations for the nonmagnetic phase of the $t-t^{\prime}$ Hubbard model, Phys. Rev. B 78, 041101(R) (2008).

[41] P. Horsch and P. Fulde, On the theory of electronic correlations in solids, Z. Phys. B 36, 23 (1979).

[42] This is strictly valid only in the absence of symmetry breaking, but the argument should remain valid as long as $\Delta_{0} \ll U$.

[43] E. Jeckelmann and D. Baeriswyl, The metal-insulator transition in polyacetylene: Variational study of the Peierls-Hubbard model, Synth. Met. 65, 211 (1994). 
[44] W. Metzner and D. Vollhardt, Ground state energies of the $\mathrm{d}=1,2,3$ dimensional Hubbard model in the weak-coupling limit, Phys. Rev. B 39, 4462 (1989).

[45] K. Ido, T. Ohgoe, and M. Imada, Competition among various charge-inhomogeneous states and $d$-wave superconducting states in Hubbard models on square lattices, Phys. Rev. B 97, 045138 (2018).

[46] From now on the symbol $\Delta_{0}$ will be used for the optimized gap parameter.

[47] J. Bardeen, L. N. Cooper, and J. R. Schrieffer, Theory of superconductivity, Phys. Rev. 108, 1175 (1957).

[48] J. R. Schrieffer, Theory of Superconductivity (Benjamin, New York, 1964).

[49] M. Sigrist and K. Ueda, Phenomenological theory of unconventional superconductivity, Rev. Mod. Phys. 63, 239 (1991).

[50] G. R. Stewart, Unconventional superconductivity, Adv. Phys. 66, 75 (2017).

[51] Y. Yanase and M. Ogata, Kinetic energy, condensation energy, optical sum rule and pairing mechanism in high- $T_{c}$ cuprates, J. Phys. Soc. Jpn. 74, 1534 (2005).

[52] Th. A. Maier, M. Jarrell, A. Macridin, and C. Slezak, Kinetic Energy Driven Pairing in Cuprate Superconductors, Phys. Rev. Lett. 92, 027005 (2004).

[53] E. Gull and A. J. Millis, Energetics of superconductivity in the two-dimensional Hubbard model, Phys. Rev. B 86, 241106(R) (2012).

[54] L. F. Tocchio, F. Becca, and S. Sorella, Hidden Mott transition and large- $U$ superconductivity in the two-dimensional Hubbard model, Phys. Rev. B 94, 195126 (2016).

[55] M. Ogata, H. Yokoyama, Y. Yanase, Y. Tanaka, and H. Tsuchiura, Kinetic-energy pairing and condensation energy in cuprates, J. Phys. Chem. Solids 67, 37 (2006).

[56] J. E. Hirsch, The true colors of cuprates, Science 295, 2226 (2002).

[57] L. Fratino, P. Sémon, G. Sordi, and A.-M. S. Tremblay, An organizing principle for two-dimensional strongly correlated superconductivity, Sci. Rep. 6, 22715 (2016).
[58] D. J. Scalapino, The case for $d_{x^{2}-y^{2}}$ pairing in the cuprate superconductors, Phys. Rep. 250, 329 (1995).

[59] J. Kondo, Superconductivity in the two-dimensional Hubbard model with a small $U$, J. Phys. Soc. Jpn. 70, 808 (2001).

[60] S. Raghu, S. A. Kivelson, and D. J. Scalapino, Superconductivity in the repulsive Hubbard model: An asymptotically exact weak-coupling solution, Phys. Rev. B 81, 224505 (2010).

[61] L. Fratino, P. Sémon, M. Charlebois, G. Sordi, and A.-M. S. Tremblay, Signatures of the Mott transition in the antiferromagnetic state of the two-dimensional Hubbard model, Phys. Rev. B 95, 235109 (2017).

[62] T. Schauerte and P. G. J. van Dongen, Symmetry breaking in the Hubbard model at weak coupling, Phys. Rev. B 65, 081105(R) (2002).

[63] R. Shankar, Renormalization-group approach to interacting fermions, Rev. Mod. Phys. 66, 129 (1994).

[64] F. B. Kugler and J. von Delft, Multiloop Functional Renormalization Group That Sums Up All Parquet Diagrams, Phys. Rev. Lett. 120, 057403 (2018).

[65] A. Tagliavini, C. Hille, F. B. Kugler, S. Andergassen, A. Toschi, and C. Honerkamp, Multiloop functional renormalization group for the two-dimensional Hubbard model: Loop convergence of the response functions, SciPost. Phys. 6, 009 (2019).

[66] P. W. Anderson, P. A. Lee, M. Randeria, T. M. Rice, N. Trivedi, and F. C. Zhang, The physics behind high-temperature superconducting cuprates: the "plain vanilla" version of RVB, J. Phys.: Condens. Matter 16, R755 (2004).

[67] D. Baeriswyl, D. Eichenberger, and M. Menteshashvili, Variational ground states of the two-dimensional Hubbard model, New J. Phys. 11, 075010 (2009).

[68] J. von Delft and D. C. Ralph, Spectroscopy of discrete energy levels in ultrasmall metallic grains, Phys. Rep. 345, 61 (2001).

[69] M. Menteshashvili (unpublished).

[70] P. W. Anderson, Theory of dirty superconductors, J. Phys. Chem. Solids 11, 26 (1959). 